J. Inst. Math. Jussieu (2023), 22(2), 879-917

doi:10.1017/S1474748021000323 C The Author(s), 2021. Published by Cambridge University

Press. This is an Open Access article, distributed under the terms of the Creative Commons

Attribution licence (http://creativecommons.org/licenses/by/4.0), which permits unrestricted re-use,

distribution and reproduction, provided the original article is properly cited

\title{
NORMAL REFLECTION SUBGROUPS OF COMPLEX REFLECTION GROUPS
}

\author{
CARLOS E. ARRECHE (D) ${ }^{1}$ AND NATHAN F. WILLIAMS (D) 2 \\ ${ }^{1}$ The University of Texas at Dallas \\ (arreche@utdallas.edu) \\ 2 The University of Texas at Dallas \\ (Nathan.Williams1@utdallas.edu)
}

(Received 3 October 2020; revised 9 June 2021; accepted 10 June 2021; first published online 21 July 2021)

\begin{abstract}
We study normal reflection subgroups of complex reflection groups. Our approach leads to a refinement of a theorem of Orlik and Solomon to the effect that the generating function for fixed-space dimension over a reflection group is a product of linear factors involving generalised exponents. Our refinement gives a uniform proof and generalisation of a recent theorem of the second author.
\end{abstract}

Keywords and Phrases: reflection groups, invariant theory, normal reflection subgroups, Poincaré series 2020 Mathematics Subject Classification: Primary Primary 20F55

Secondary 05E10

\section{Introduction}

\subsection{Lie Groups}

Hopf proved that the cohomology of a real connected compact Lie group $\mathcal{G}$ is a free exterior algebra on $r=\operatorname{rank}(\mathcal{G})$ generators of odd degree [12]. Its Poincaré series is therefore given by

$$
\operatorname{Hilb}\left(H^{*}(\mathcal{G}) ; q\right)=\prod_{i=1}^{r}\left(1+q^{2 e_{i}+1}\right) .
$$

Chevalley presented these $e_{i}$ for the exceptional simple Lie algebras in his 1950 address at the International Congress of Mathematicians [8], and Coxeter recognised them from previous work with real reflection groups [10]. This observation has led to deep relationships between the cohomology of $\mathcal{G}$ and the invariant theory of the corresponding Weyl group $W=N_{\mathcal{G}}(T) / T$, where $T$ is a maximal torus in $\mathcal{G}[18,17]$ - notably,

$$
H^{*}(\mathcal{G}) \simeq\left(H^{*}(\mathcal{G} / T) \times H^{*}(T)\right)^{W} \simeq\left(S\left(V^{*}\right) / I_{W}^{+} \otimes \bigwedge V^{*}\right)^{W}
$$


where $V=\operatorname{Lie}(T)$ is the reflection representation of $W, S\left(V^{*}\right)$ is the algebra of polynomial functions on $V$ and $I_{W}^{+}$is the ideal generated by the $W$-invariant polynomials in $S\left(V^{*}\right)$ with no constant term. For more details, we refer the reader to the wonderful survey [3].

\subsection{Complex Reflection Groups}

It turns out that the $e_{i}$ in Equation (1) can be computed from the generating function for the dimension of the fixed $\operatorname{space} \operatorname{fix}(w):=\operatorname{dim}(\operatorname{ker}(1-w))$ for $w \in W$, via the remarkable formula:

$$
\sum_{w \in W} q^{\mathrm{fix}(w)}=\prod_{i=1}^{r}\left(q+e_{i}\right)
$$

Shephard and Todd verified case by case that the same sum still factors when $W$ is replaced by a finite complex reflection group $G \subset \mathrm{GL}(V)$ acting by reflections on a complex vector space $V$ of dimension $r$ [19, Theorem 5.3]. The $e_{i}$ are now determined by the degrees $d_{i}$ of the fundamental invariants of $G$ on $V$ as $e_{i}=d_{i}-1$. A case-free proof of this result was given by Solomon in [20], mirroring Hopf's result: $\left(S\left(V^{*}\right) \otimes \wedge V^{*}\right)^{G}$ is a free exterior algebra over the $\operatorname{ring} S\left(V^{*}\right)^{G}$ of $G$-invariant polynomials, which gives a factorisation of the Poincaré series of the $G$-invariant differential forms

$$
\operatorname{Hilb}\left(\left(S\left(V^{*}\right) \otimes \bigwedge V^{*}\right)^{G} ; q, u\right)=\prod_{i=1}^{r} \frac{1+u q^{e_{i}}}{1-q^{d_{i}}}
$$

Computing the trace of the projection $\frac{1}{|G|} \sum_{g \in G} g$ to the subspace of $G$-invariants on $S\left(V^{*}\right) \otimes \bigwedge V^{*}$, specialising to $u=q(1-x)-1$ and taking the limit as $x \rightarrow 1$ gives the Shephard-Todd result in Equation (2).

\subsection{Galois twists and cohomology}

More generally, the fake degree of an $m$-dimensional simple $G$-module $M$ is the polynomial encoding the degrees in which $M$ occurs in the coinvariant algebra $S\left(V^{*}\right) / I_{G}^{+} \simeq \mathcal{C}_{G}$ :

$$
f_{M}(q)=\sum_{i}\left(\left(\mathcal{C}_{G}\right)_{i}, M\right) q^{i}=\sum_{i=1}^{m} q^{e_{i}(M)}
$$

The fake degree of a reducible $G$-module is defined as the sum of the fake degrees of its simple direct summands. The integers $e_{i}(M)$ in Equation (4) are called the $M$-exponents of $G$.

Letting $\zeta_{G}$ denote a primitive $|G|$ th root of unity, for $\sigma \in \operatorname{Gal}\left(\mathbb{Q}\left(\zeta_{G}\right) / \mathbb{Q}\right)$ the Galois twist $V^{\sigma}$ is the representation of $G$ obtained by applying $\sigma$ to its matrix entries. In [16], Orlik and Solomon gave a beautiful generalisation of Equations (2) and (3) that takes into account these Galois twists (see Section 2.3). 
Theorem 1.1 ([16, Thm. 3.3]). Let $G \subset \mathrm{GL}(V)$ be a complex reflection group of rank $r$ and let $\sigma \in \operatorname{Gal}\left(\mathbb{Q}\left(\zeta_{G}\right) / \mathbb{Q}\right)$. Then

$$
\sum_{g \in G}\left(\prod_{\lambda_{i}(g) \neq 1} \frac{1-\lambda_{i}(g)^{\sigma}}{1-\lambda_{i}(g)}\right) q^{\mathrm{fix}_{V}(g)}=\prod_{i=1}^{r}\left(q+e_{i}\left(V^{\sigma}\right)\right),
$$

where the $\lambda_{i}(g)$ are the eigenvalues of $g \in G$ acting on $V$.

When $\sigma: \zeta_{G} \mapsto \overline{\zeta_{G}}$ is complex conjugation, Orlik and Solomon [16, Thm. 4.8] further connected their Theorem 1.1 to the cohomology of the complement of the corresponding hyperplane arrangement - in this case $V^{\sigma} \simeq V^{*}$ as a $G$-representation, and the coexponents $e_{i}\left(V^{*}\right)$ are the degrees of the generators of the cohomology ring of the complement of the hyperplane arrangement.

\subsection{Normal Reflection Subgroups of Complex Reflection Groups}

Let $G \subset \mathrm{GL}(V)$ be a complex reflection group. We say that $N \unlhd G$ is a normal reflection subgroup of $G$ if it is a normal subgroup of $G$ that is generated by reflections. The main theorem of this article, Theorem 1.4, gives a new refinement of Theorem 1.1 to accommodate a normal reflection subgroup. The following result is a special case of [4], where they consider the more general notion of bon sous-groupe distingué in lieu of our normal reflection subgroup $N$ of $G$.

Theorem 1.2. Let $G \subset \mathrm{GL}(V)$ be a complex reflection group and let $N \unlhd G$ be a normal reflection subgroup. Then $G / N=H$ acts as a reflection group on the vector space $V / N=E$.

The bons sous-groupes distingués of [4] are precisely those normal subgroups for which the associated quotient group is a reflection group acting on the tangent space at 0 of $V / N$, which is a strictly weaker condition than being a normal reflection subgroup. Our proof of Theorem 1.2 in Section 3 follows the ideas of [4] but specialised to our more restricted setting where the normal subgroup under consideration is actually a normal reflection subgroup. In this more restricted setting, we are able to prove the new results Theorems 1.3 and 1.4 stated below.

The technical definition of the $G$-module $U_{\sigma}^{N}$ that mediates the statement of the following result is given in Definition 2.9. Because we are dealing with multiple reflection groups acting on multiple spaces, we will begin labelling exponents and degrees by their corresponding groups.

Theorem 1.3. Let $G \subset \mathrm{GL}(V)$ be a complex reflection group and let $N \unlhd G$ be a normal reflection subgroup. Let $H=G / N$ and $E=V / N$. Then for a suitable choice of indexing we have

$$
\begin{aligned}
e_{i}^{N}\left(V^{\sigma}\right)+e_{i}^{G}\left(U_{\sigma}^{N}\right) & =e_{i}^{G}\left(V^{\sigma}\right) \\
d_{i}^{N} \cdot e_{i}^{H}\left(E^{\sigma}\right) & =e_{i}^{G}\left(E^{\sigma}\right) \\
d_{i}^{N} \cdot d_{i}^{H} & =d_{i}^{G} .
\end{aligned}
$$


In the special case $\sigma=1$, it is well known that $U_{\sigma}^{N} \simeq E$ as $G$-modules (see Definition 2.9 and Lemma 4.1), so that Theorem 1.3 coincides with [1, Theorem 1.3] in this case. As we explain in Remark 4.2, in this special case where $\sigma=1$, the equalities in Theorem 1.3 are compatible with the relations $d_{i}=e_{i}+1$ between classical exponents and degrees for the three reflection groups involved.

An essential tool in our proof of Theorem 1.3 is Proposition 3.3, which gives a graded $G$-module isomorphism $\mathcal{C}_{G} \simeq \mathcal{C}_{H} \otimes \mathcal{C}_{N}$ relating the spaces of harmonic polynomials for $N$ and $H$ to that for $G$, which is an interesting and useful result in its own right.

Our Theorem 1.4 generalises the Orlik-Solomon formula from Theorem 1.1 to take into account the additional combinatorial data arising from a normal reflection subgroup. The technical definition of the $G$-module $U_{\sigma}^{N}$ is again given in Definition 2.9.

Theorem 1.4. Let $G \subset \mathrm{GL}(V)$ be a complex reflection group of rank $r$ and let $N \unlhd G$ be a normal reflection subgroup. Let $E=V / N$ and $\sigma \in \operatorname{Gal}\left(\mathbb{Q}\left(\zeta_{G}\right) / \mathbb{Q}\right)$. Then for a suitable choice of indexing we have

$$
\sum_{g \in G}\left(\prod_{\lambda_{i}(g) \neq 1} \frac{1-\lambda_{i}(g)^{\sigma}}{1-\lambda_{i}(g)}\right) q^{\mathrm{fix}_{V}(g)} t^{\mathrm{fix}_{E}(g)}=\prod_{i=1}^{r}\left(q t+e_{i}^{N}\left(V^{\sigma}\right) t+e_{i}^{G}\left(U_{\sigma}^{N}\right)\right)
$$

where the $\lambda_{i}(g)$ are the eigenvalues of $g \in G$ acting on $V$.

In view of Theorem 1.3, specialising to $t=1$ recovers Theorem 1.1. Moreover, because $U_{\sigma}^{N} \simeq E$ as $G$-modules when $\sigma=1$ (see again Definition 2.9 and Lemma 4.1), Theorem 1.4 coincides with $[1$, Theorem 1.5$]$ in this case, which when similarly specialised to $t=1$ recovers Equation (2). As explained in Remark 4.12, one can also recover Theorem 1.1 for the reflection group $N$ from Theorem 1.4 by applying $\frac{1}{r !} \frac{\partial^{r}}{\partial t^{r}}$ on both sides. In the special case $\sigma=1$, one can recover Equation (2) for the reflection group $H$ by specialising Theorem 1.4 to $q=1$ and dividing by $|N|$ on both sides, but this same specialisation does not seem to be directly related to Theorem 1.1 for $H$ in general for arbitrary $\sigma \in$ $\operatorname{Gal}\left(\mathbb{Q}\left(\zeta_{G}\right) / \mathbb{Q}\right)$.

Our proof of Theorem 1.4 follows a strategy similar to the one employed in [16]: we compute the Poincaré series for $\left(S\left(V^{*}\right) \otimes \bigwedge\left(U_{\sigma}^{N}\right)^{*}\right)^{G}$ in two equivalent and standard ways and then obtain Theorem 1.4 from a well-chosen specialisation. However, a delicate technical issue arises in that our specialisation does not provide the correct contribution term by term in the left-hand side of Theorem 1.4. We overcome this technical difficulty by applying the results of [7], where the authors develop a 'twisted invariant theory' for cosets $N g$ of a reflection group $N \subset \mathrm{GL}(V)$ for $g \in \mathrm{GL}(V)$ an element of the normaliser of $N$ in GL $(V)$. Our proof of Theorem 1.4 applies the results of [7] to the special situation where the cosets $N g$ all come from $g \in G$, a reflection group containing $N$ as a normal reflection subgroup, to show that our specialisation argument does provide the correct contribution coset by coset.

In summary, we have applied results and insights from [7, 4] in the development of new results in the invariant theory for complex reflection groups $G$ taking into account the additional combinatorial data arising from normal reflection subgroups $N \unlhd G$ and their corresponding reflection group quotients $H=G / N$. The setting of [4] considers 
more general $N$ (their bons sous-groups distingués), whereas the setting of [7] considers more general cosets $N g$ (for arbitrary $g$ in the normaliser of $N$ ). The general study of normal reflection subgroups initiated in this article is both natural, because it lies in the

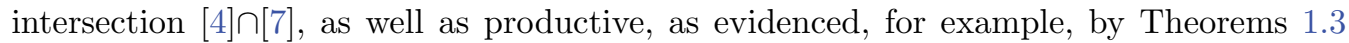
and 1.4, the graded $G$-module isomorphism $\mathcal{C}_{G} \simeq \mathcal{C}_{H} \otimes \mathcal{C}_{N}$ of Proposition 3.3 and the ancillary results in Section 3 relating the amenability of different modules with respect to the groups $G, N$, and $H$.

\subsection{Organisation}

We recall standard results about complex reflection groups in Section 2. In Section 3, we introduce normal reflection subgroups and prove ancillary results, relating spaces of harmonic polynomials and amenability with respect to different reflection groups. We prove the main results stated in the Introduction, Theorems 1.3 and 1.4, in Section 4. In Section 5 we recall the case-by-case results of [22] and discuss how they are obtained in a case-free manner by the methods of the present article. In Section 6 we provide a complete classification of the normal reflection subgroups of the irreducible complex reflection groups. Finally, in Section 7 we give several examples that illustrate our general results.

\section{Invariant Theory of Reflection Groups}

Let $V$ be a complex vector space of dimension $r$. A reflection is an element of $\operatorname{GL}(V)$ of finite order that fixes some hyperplane pointwise. A complex reflection group $G$ is a finite subgroup of GL $(V)$ that is generated by reflections. A complex reflection group $G$ is called irreducible if $V$ is a simple $G$-module; $V$ is then called the reflection representation of $G$. A (normal) reflection subgroup of $G$ is a (normal) subgroup that is generated by reflections. In what follows, a $G$-module will always be a complex representation of $G$.

\subsection{Chevalley-Shephard-Todd's Theorem}

Let $S\left(V^{*}\right)$ be the symmetric algebra on the dual vector space $V^{*}$, and write $S\left(V^{*}\right)^{G}$ for its $G$-invariant subring. By a classical theorem of Shephard-Todd [19] and Chevalley [9], a finite subgroup $G \subset \mathrm{GL}(V)$ is a complex reflection group if and only if $S\left(V^{*}\right)^{G}$ is a polynomial ring, and in this case $S\left(V^{*}\right)^{G}$ is generated by $r$ algebraically independent homogeneous polynomials - the degrees $d_{1} \leq \cdots \leq d_{r}$ of these polynomials are invariants of $G$.

Theorem 2.1 ([9, 19]). A finite subgroup $G \subset \mathrm{GL}(V)$ is a complex reflection group if and only if there exist $r=\operatorname{dim}(V)$ homogeneous algebraically independent polynomials $G_{1}, \ldots, G_{r}$ such that $S\left(V^{*}\right)^{G}=\mathbb{C}\left[G_{1}, \ldots, G_{r}\right]$. In this case, $|G|=\prod_{i=1}^{r} d_{i}$, where $d_{i}=$ $\operatorname{deg}\left(G_{i}\right)$.

Let $I_{G}^{+} \subset S\left(V^{*}\right)$ denote the ideal generated by homogeneous $G$-invariant polynomials of positive degree. In [9], Chevalley proved that, as an ungraded $G$-module, $S\left(V^{*}\right) / I_{G}^{+}$ affords the regular representation of $G$. Because $I_{G}^{+}$is $G$-stable, we may choose a 
$G$-stable complement $\mathcal{C}_{G} \subset S\left(V^{*}\right)$, so that $S\left(V^{*}\right) \simeq I_{G}^{+} \oplus \mathcal{C}_{G}$ as graded $G$-modules, and $\mathcal{C}_{G}$ is a graded version of the regular representation of $G$. Chevalley also proved in [9] that $S\left(V^{*}\right) \simeq S\left(V^{*}\right)^{G} \otimes \mathcal{C}_{G}$ as graded $G$-modules. A canonical choice for such a $G$-stable complement $\mathcal{C}_{G}$ is the space of $G$-harmonic polynomials [14, Corollary 9.37]; that is, polynomials in $S\left(V^{*}\right)$ that are annihilated by all $G$-invariant polynomial differential operators with no constant term [14, Definition 9.35].

Remark 2.2. The space $\mathcal{C}_{G}$ of $G$-harmonic polynomials is stabilised by the normaliser of $G$ in $\operatorname{GL}(V)$ [14, Proposition 12.2]. This fact will be essential in our treatment of normal reflection subgroups.

Our choice of notation $\mathcal{C}$ for the space of harmonic polynomials, instead of the more common and natural $\mathcal{H}$ used in the literature, is meant to avoid unfortunate phonetic confusion with the quotient group $H=G / N$ that will play a prominent role in the rest of the article.

\subsection{Solomon's Theorem}

We recall the following celebrated theorem of Solomon.

Theorem 2.3 ([20]). $\left(S\left(V^{*}\right) \otimes \wedge V^{*}\right)^{G}$ is a free exterior algebra over the ring of $G$ invariant polynomials

$$
\left(S\left(V^{*}\right) \otimes \bigwedge V^{*}\right)^{G} \simeq S\left(V^{*}\right)^{G} \otimes \bigwedge\left(U^{G}\right)^{*}
$$

where $\left(U^{G}\right)^{*}=\operatorname{span}_{\mathbb{C}}\left\{d G_{1}, \ldots, d G_{r}\right\}$ and $d G_{i}=\sum_{j=1}^{r} \frac{\partial G_{i}}{\partial x_{j}} \otimes x_{j}$ form a free basis for $\left(S\left(V^{*}\right) \otimes V^{*}\right)^{G}$ over $S\left(V^{*}\right)^{G}$.

Computing the trace on $S\left(V^{*}\right) \otimes \bigwedge V^{*}$ of the projection to the $G$-invariants $\frac{1}{|G|} \sum_{g \in G} g$ gives a formula for the Poincaré series as a sum over the group.

Corollary 2.4 ([20]).

$$
\operatorname{Hilb}\left(\left(S\left(V^{*}\right) \otimes \bigwedge V^{*}\right)^{G} ; x, u\right)=\frac{1}{|G|} \sum_{g \in G} \frac{\operatorname{det}\left(1+\left.u g\right|_{V}\right)}{\operatorname{det}\left(1-\left.x g\right|_{V}\right)}=\prod_{i=1}^{r} \frac{1+x^{e_{i}^{G}(V)} u}{1-x_{i}^{d_{i}^{G}}} .
$$

Specialising Corollary 2.4 to $u=q(1-x)-1$ and taking the limit as $x \rightarrow 1$ gives the Shephard-Todd formula from Equation (2).

\subsection{Orlik-Solomon's Theorem}

The reflection representation $V$ of $G \subset \mathrm{GL}(V)$ can be realised over $\mathbb{Q}\left(\zeta_{G}\right)$, where $\zeta_{G}$ denotes a primitive $|G|$ th root of unity, in the sense that there is a choice of basis for $V$ with respect to which $G \subset \mathrm{GL}_{r}\left(\mathbb{Q}\left(\zeta_{G}\right)\right)$. For $\sigma \in \operatorname{Gal}\left(\mathbb{Q}\left(\zeta_{G}\right) / \mathbb{Q}\right)$, the Galois twist $V^{\sigma}$ of $V$ is the representation of $G$ on the same underlying vector space $V$ obtained by applying $\sigma$ to the matrix entries of $g \in \mathrm{GL}_{r}\left(\mathbb{Q}\left(\zeta_{G}\right)\right)$. Alternatively and equivalently, one can define $V^{\sigma}$ by applying $\tilde{\sigma}$ to the matrix entries of $g$ in terms of any basis of $V$, for $\tilde{\sigma}$ any extension of $\sigma$ to a field automorphism of $\mathbb{C}$.

In [16], Orlik and Solomon gave the following generalisation of Theorem 2.3. 
Theorem 2.5 ([16, Corollary 3.2]).

$$
\left(S\left(V^{*}\right) \otimes \bigwedge\left(V^{\sigma}\right)^{*}\right)^{G} \simeq S\left(V^{*}\right)^{G} \otimes \bigwedge\left(U_{\sigma}^{G}\right)^{*}
$$

where the degrees of the homogeneous generators of $\left(U_{\sigma}^{G}\right)^{*}:=\left(\mathcal{C}_{G} \otimes\left(V^{\sigma}\right)^{*}\right)^{G}$ are $e_{i}^{G}\left(V^{\sigma}\right)$, the $V^{\sigma}$-exponents of $G$.

Computing the Poincaré series in two ways as in Corollary 2.4 gives the following formula.

Corollary 2.6 ([16, Theorem 3.3]).

$$
\operatorname{Hilb}\left(\left(S\left(V^{*}\right) \otimes \bigwedge\left(V^{\sigma}\right)^{*}\right)^{G} ; x, u\right)=\frac{1}{|G|} \sum_{g \in G} \frac{\operatorname{det}\left(1+\left.u g\right|_{V^{\sigma}}\right)}{\operatorname{det}\left(1-\left.x g\right|_{V}\right)}=\prod_{i=1}^{r} \frac{1+x^{e_{i}^{G}\left(V^{\sigma}\right)} u}{1-x_{i}^{d_{i}^{G}}} .
$$

Specialising Corollary 2.6 to $u=q(1-x)-1$ and taking the limit as $x \rightarrow 1$ gives Theorem 1.1.

\subsection{Amenable Representations}

More generally, an $m$-dimensional $G$-module $M$ satisfying $\sum_{i=1}^{m} e_{i}^{G}(M)=e_{1}^{G}\left(\bigwedge^{m} M\right)$ is called amenable. This amenability condition can be shown to be equivalent to the requirement that $\left(S\left(V^{*}\right) \otimes \bigwedge M^{*}\right)^{G}$ be a free exterior algebra over $S\left(V^{*}\right)^{G}$. Because, in particular, Galois twists $V^{\sigma}$ of the reflection representation $V$ of $G$ are amenable, the following theorem generalises Theorem 2.5.

Theorem 2.7 ([16, Theorem 3.1]). Let $M$ be an amenable G-module. Then

$$
\left(S\left(V^{*}\right) \otimes \bigwedge M^{*}\right)^{G} \simeq S\left(V^{*}\right)^{G} \otimes \bigwedge\left(U_{M}^{G}\right)^{*}
$$

where $\left(U_{M}^{G}\right)^{*}:=\left(\mathcal{C}_{G} \otimes M^{*}\right)^{G}$ and the degrees of the homogeneous generators of $\left(U_{M}^{G}\right)^{*}$ are $e_{i}^{G}(M)$, the $M$-exponents of $G$.

From this, one can pursue the usual strategy of computing the Poincaré series of $\left(S\left(V^{*}\right) \otimes \bigwedge M^{*}\right)^{G}$ in two different ways to obtain the following.

Corollary 2.8. If $M$ is an amenable G-module, then

$$
\operatorname{Hilb}\left(\left(S\left(V^{*}\right) \otimes \bigwedge M^{*}\right)^{G} ; x, u\right)=\frac{1}{|G|} \sum_{g \in G} \frac{\operatorname{det}\left(1+\left.u g\right|_{M}\right)}{\operatorname{det}\left(1-\left.x g\right|_{V}\right)}=\frac{\prod_{i=1}^{m}\left(1+x^{e_{i}^{G}(M)} u\right)}{\prod_{i=1}^{r}\left(1-x_{i}^{d_{i}^{G}}\right)} .
$$

However, it is no longer clear how to specialise Corollary 2.8 in the same way as Corollaries 2.4 and 2.6 to obtain an analogue of Equation (2) and Theorem 1.1 in this generality.

Definition 2.9. Let $M$ be a $G$-module. We define the Orlik-Solomon space $U_{M}^{G}$ to be the dual $G$-module to $\left(U_{M}^{G}\right)^{*}:=\left(\mathcal{C}_{G} \otimes M^{*}\right)^{G}$. In the special case where $M=V$, we write $U^{G}:=U_{V}^{G}$. When $M=V^{\sigma}$, we write $U_{\sigma}^{G}:=U_{V^{\sigma}}^{G}$. 


\section{Normal Reflection Subgroups}

The following theorem is a special case of results in [4] (where they consider the more general notion of bon sous-groupe distingué in lieu of our normal reflection subgroup $N$ of $G)$. We emphasise that our proof follows the ideas in [4], specialised to our more restricted setting.

Theorem 1.2. Let $G \subset \mathrm{GL}(V)$ be a complex reflection group and let $N \unlhd G$ be a normal reflection subgroup. Then $G / N=H$ acts as a reflection group on the vector space $V / N=E$.

Proof. We claim that there exist homogeneous generators $N_{1}, \ldots, N_{r}$ of $S\left(V^{*}\right)^{N}$ such that $E^{*}=\operatorname{span}_{\mathbb{C}}\left\{N_{1}, \ldots, N_{r}\right\}$ is $H$-stable. By Theorem 2.1, the ring of $N$-invariants $S\left(V^{*}\right)^{N}=$ $\mathbb{C}\left[\tilde{N}_{1}, \ldots, \tilde{N}_{r}\right]$ for some homogeneous algebraically independent $\tilde{N}_{i}$. Let $I_{+} \subset S\left(V^{*}\right)^{N}$ be the ideal generated by homogeneous $N$-invariants of positive degree. Then both $I_{+}$and $I_{+}^{2}$ are $H$-stable homogeneous ideals, and therefore the algebraic tangent space $I_{+} / I_{+}^{2}$ to $E=V / N$ at 0 inherits a graded action of $H$ that is compatible with the (graded) quotient map $\pi: I_{+} \rightarrow I_{+} / I_{+}^{2}$. Hence, there exists a graded $H$-equivariant section $\varphi: I_{+} / I_{+}^{2} \rightarrow$ $I_{+}$. Letting $N_{i}=\varphi \circ \pi\left(\tilde{N}_{i}\right)$, we see that $N_{1}, \ldots, N_{r}$ are still homogeneous algebraically independent generators for $S\left(V^{*}\right)^{N}$ with $\operatorname{deg}\left(N_{i}\right)=\operatorname{deg}\left(\tilde{N}_{i}\right)$ and $E^{*}:=\operatorname{span}_{\mathbb{C}}\left\{N_{1}, \ldots, N_{r}\right\}$ is $H$-stable.

Let $\mathbf{x}=\left\{x_{1}, \ldots, x_{r}\right\}$ denote a dual basis for $V$ and $\mathbf{N}=\left\{N_{1}, \ldots, N_{r}\right\}$ denote an $H$ stable basis for $E^{*}$ as above. Because the action of $H$ on the polynomial $\operatorname{ring} S\left(E^{*}\right)=$ $S\left(V^{*}\right)^{N}$ is obtained from the action of $G$ on $S\left(V^{*}\right)$, it preserves $\mathbf{x}$-degrees as well as $\mathbf{N}$-degrees. Therefore, we may choose the fundamental $G$-invariants $G_{i}(\mathbf{x}) \in S\left(V^{*}\right)^{G}=$ $\left(S\left(V^{*}\right)^{N}\right)^{H}=S\left(E^{*}\right)^{H}$ to be simultaneously $\mathbf{x}$-homogeneous and $\mathbf{N}$-homogeneous, so that $H_{i}(\mathbf{N}):=G_{i}(\mathbf{x})$ form a set of $\mathbf{N}$-homogeneous generators for the polynomial ring $S\left(E^{*}\right)^{H}$. Because any algebraic relation $f\left(H_{1}, \ldots, H_{r}\right)=0$ would result in an algebraic relation $f\left(G_{1}, \ldots, G_{r}\right)=0$, the $\mathbf{N}$-homogeneous $H_{i}(\mathbf{N})$ must be algebraically independent. By Theorem 2.1, $H$ is a complex reflection group.

Remark 3.1. As pointed out in [4, Proposition 3.16] and explained in [7, Section 8.3], the action of $H$ on $E$ is often not irreducible. Denote by $\mathcal{D}_{N}=\left\{d_{1}^{N}, \ldots, d_{r}^{N}\right\}$ the set of degrees $d_{i}^{N}:=\operatorname{deg}_{\mathbf{x}}\left(N_{i}\right)$. For $d \in \mathcal{D}_{N}$, let us write $\mathbf{N}_{d}:=\left\{N_{i} \in \mathbf{N} \mid \operatorname{deg}_{\mathbf{x}}\left(N_{i}\right)=d\right\}$ and $E_{d}^{*}:=\operatorname{span}_{\mathbb{C}} \mathbf{N}_{d}$, so that $E^{*} \simeq \bigoplus_{d \in \mathcal{D}_{N}} E_{d}^{*}$ and $S\left(E^{*}\right) \simeq \bigotimes_{d \in \mathcal{D}_{N}} S\left(E_{d}^{*}\right)$ as graded $H$ modules. For $d \in \mathcal{D}_{N}$, let $H_{(d)} \subset \mathrm{GL}\left(E_{d}^{*}\right)$ denote the image of $H$ in $\operatorname{GL}\left(E_{d}^{*}\right)$, so that $H$ decomposes as a direct product $\chi_{d \in \mathcal{D}_{N}} H_{(d)}$, where each $H_{(d)}$ is a reflection group on the graded dual $E_{-d}$ of $E_{d}^{*}$. We see that in fact there exist algebraically independent (bi)homogeneous polynomials $H_{i}\left(\mathbf{N}_{d_{i}^{N}}\right) \in S\left(E_{d_{i}^{N}}^{*}\right)$ such that $S\left(E^{*}\right)^{H}=\mathbb{C}\left[H_{1}, \ldots, H_{r}\right]$, so that the fundamental $G$-invariants $G_{1}, \ldots, G_{r}$ generating $S\left(V^{*}\right)^{G}=\left(S\left(V^{*}\right)^{N}\right)^{H}=S\left(E^{*}\right)^{H}$ can be expressed as

$$
G_{i}(\mathbf{x})=H_{i}\left(\mathbf{N}_{d_{i}^{N}}\right)
$$

Having chosen the fundamental $G$-invariants $G_{i}$ to have degrees $d_{1}^{G} \leq \cdots \leq d_{r}^{G}$, we implicitly index the $N$-degrees $d_{i}^{N}$ and $H$-degrees $d_{i}^{H}$ so that Equation (5) is satisfied. 
Remark 3.2. Unlike in the real case $[5,11], H$ is not necessarily (isomorphic to) a reflection subgroup of $G$ or even a subgroup of $G$. A counterexample is given by $G_{8}=$ $G \triangleright N=G(4,2,2)$, so that $G / N \simeq \mathfrak{S}_{3}-$ but $\mathfrak{S}_{3}$ is not a subgroup of $G_{8}$.

\subsection{Harmonic polynomials}

The space of $N$-harmonic polynomials $\mathcal{C}_{N} \subset S\left(V^{*}\right)$ is $G$-stable [14, Proposition 12.2] and isomorphic to the regular representation of $N$ [14, Corollary 9.37]. The space of $H$-harmonic polynomials $\mathcal{C}_{H} \subset S\left(E^{*}\right)$ is bigraded, by $\mathbf{x}$-degree as well as by $\mathbf{N}$-degree, and therefore it admits a $\mathbb{C}$-basis of $H$-harmonic polynomials that are simultaneously $\mathbf{x}$ homogeneous and $\mathbf{N}$-homogeneous. The following result elaborates on [7, Corollary 8.4] in our present setting.

Proposition 3.3. There is a graded G-module isomorphism $\mathcal{C}_{G} \simeq \mathcal{C}_{H} \otimes \mathcal{C}_{N}$ such that $\left(\mathcal{C}_{G}\right)^{N} \simeq \mathcal{C}_{H}$ as graded H-modules.

Proof. Putting together the isomorphisms $S\left(V^{*}\right) \simeq S\left(E^{*}\right) \otimes \mathcal{C}_{N}$ as graded $N$-modules, $S\left(E^{*}\right) \simeq S\left(E^{*}\right)^{H} \otimes \mathcal{C}_{H}$ as bigraded $H$-modules (equivalently, as bigraded $G$-modules of $N$ invariants) and $S\left(V^{*}\right)^{G}=S\left(E^{*}\right)^{H}$, we obtain the isomorphism $S\left(V^{*}\right) \simeq S\left(V^{*}\right)^{G} \otimes \mathcal{C}_{H} \otimes \mathcal{C}_{N}$ as graded $G$-modules. Letting $\pi: S\left(V^{*}\right) \rightarrow S\left(V^{*}\right) / I_{G}^{+}$denote the canonical projection, we see that $\mathbb{C} \otimes \mathcal{C}_{H} \otimes \mathcal{C}_{N}$ must surject onto the image $S\left(V^{*}\right) / I_{G}^{+} \simeq \mathcal{C}_{G}$, because $S\left(V^{*}\right)^{G}$ is generated as a $\mathbb{C}$-algebra by the generators of the ideal $I_{G}^{+}$. But this surjection $\mathcal{C}_{H} \otimes \mathcal{C}_{N} \rightarrow$ $\mathcal{C}_{G}$ of graded $G$-modules must then be an isomorphism, because

$$
\operatorname{dim}_{\mathbb{C}}\left(\mathcal{C}_{H} \otimes \mathcal{C}_{N}\right)=\operatorname{dim}_{\mathbb{C}}\left(\mathcal{C}_{H}\right) \cdot \operatorname{dim}_{\mathbb{C}}\left(\mathcal{C}_{N}\right)=|H| \cdot|N|=|G|=\operatorname{dim}_{\mathbb{C}}\left(\mathcal{C}_{G}\right) .
$$

Because $\mathcal{C}_{H} \subset S\left(E^{*}\right)$ consists of $N$-invariants, we have $\left(\mathcal{C}_{H} \otimes \mathcal{C}_{N}\right)^{N}=\mathcal{C}_{H} \otimes\left(\mathcal{C}_{N}\right)^{N}=\mathcal{C}_{H} \otimes$ $\mathbb{C}$, and therefore $\left(\mathcal{C}_{G}\right)^{N} \simeq \mathcal{C}_{H}$ as graded $H$-modules, as claimed.

Remark 3.4. It follows from the graded $G$-isomorphism $S\left(V^{*}\right) \simeq S\left(V^{*}\right)^{G} \otimes \mathcal{C}_{G}$ that the Poincaré series of $\mathcal{C}_{G}$ can be written as [9, Theorem B]

$$
\operatorname{Hilb}\left(\mathcal{C}_{G} ; q\right)=\frac{\operatorname{Hilb}\left(S\left(V^{*}\right) ; q\right)}{\operatorname{Hilb}\left(S\left(V^{*}\right)^{G} ; q\right)}=\prod_{i=1}^{r} \frac{1-q^{d_{i}^{G}}}{1-q} .
$$

Because $|G|=d_{1} \cdots d_{r}$, it is natural to ask for a combinatorial interpretation of $\operatorname{Hilb}\left(\mathcal{C}_{G} ; q\right)$ as a weighted sum over the elements of $G$. When $G$ is a real reflection group, $G$ acts simply transitively on the connected components of its real hyperplane complement. Assigning some base connected component $R_{e}$ to the identity element $e \in G$ gives a bijection between group elements and connected components of the hyperplane complement $g \leftrightarrow R_{g}$, and we can define the statistic $\operatorname{inv}(g)$ to be the number of inversions of $g \in G$; that is, the number of hyperplanes separating the connected component $R_{g}$ from $R_{e}$. In this case the Poincaré series of $\mathcal{C}_{G}$ has the well-known interpretation (see for example [5, Section 7.1] or $[13, \S 3])$

$$
\operatorname{Hilb}\left(\mathcal{C}_{G} ; q\right)=\prod_{i=1}^{r} \frac{1-q^{d_{i}^{G}}}{1-q}=\sum_{g \in G} q^{\operatorname{inv}(g)}
$$


The graded $G$-module isomorphism $\mathcal{C}_{G} \simeq \mathcal{C}_{H} \otimes \mathcal{C}_{N}$ of Proposition 3.3 yields a factorisation

$$
\operatorname{Hilb}\left(\mathcal{C}_{G} ; q\right)=\operatorname{Hilb}\left(\mathcal{C}_{H} ; q\right) \cdot \operatorname{Hilb}\left(\mathcal{C}_{N} ; q\right)
$$

(bearing in mind that the quotient group $H=G / N$ acts by reflections on the graded vector space $E=V / N$ as detailed in Remark 3.1, and $\mathcal{C}_{H}$ is endowed with the resulting grading for Equation (6) to hold). This allows us to produce a combinatorial interpretation for $\operatorname{Hilb}\left(\mathcal{C}_{G} ; q\right)$ in some additional cases: provided prior combinatorial interpretations

$$
\operatorname{Hilb}\left(\mathcal{C}_{H} ; q\right)=\sum_{h \in H} q^{\text {stat }_{H}(h)} \quad \text { and } \operatorname{Hilb}\left(\mathcal{C}_{N} ; q\right)=\sum_{n \in N} q^{\text {stat }_{N}(n)},
$$

we can then obtain an interpretation

$$
\operatorname{Hilb}\left(\mathcal{C}_{G} ; q\right)=\sum_{g \in G} q^{\text {stat }_{G}(g)}=\sum_{h \in H} q^{\text {stat }_{H}(h)}\left(\sum_{n \in N} q^{\operatorname{stat}_{N}(n)}\right) .
$$

We will now illustrate this strategy with two concrete (and scaffolded) examples.

A combinatorial interpretation for $\operatorname{Hilb}\left(\mathcal{C}_{G(4,2,2)} ; q\right)$ is obtained from the choice of normal reflection subgroup

$$
N=G(2,2,2)=\left\{\left(\begin{array}{ll}
1 & 0 \\
0 & 1
\end{array}\right),\left(\begin{array}{ll}
0 & 1 \\
1 & 0
\end{array}\right),\left(\begin{array}{cc}
0 & -1 \\
-1 & 0
\end{array}\right),\left(\begin{array}{cc}
-1 & 0 \\
0 & -1
\end{array}\right)\right\}
$$

which is real and therefore admits the combinatorial interpretation described above in terms of the inversion statistic $\operatorname{Hilb}\left(\mathcal{C}_{N} ; q\right)=1+q+q+q^{2}=(1+q)^{2}$. Let us choose the coset representatives $g_{h} \in G(4,2,2)$ for $h \in H \simeq C_{2} \times C_{2}$ to be

$$
\left\{g_{(1,1)}, g_{(-1,1)}, g_{(1,-1)}, g_{(-1,-1)}\right\}=\left\{\left(\begin{array}{ll}
1 & 0 \\
0 & 1
\end{array}\right),\left(\begin{array}{cc}
0 & i \\
i & 0
\end{array}\right),\left(\begin{array}{cc}
0 & -1 \\
1 & 0
\end{array}\right),\left(\begin{array}{cc}
i & 0 \\
0 & -i
\end{array}\right)\right\} \text {. }
$$

Then we obtain the interpretation

$$
\operatorname{Hilb}\left(\mathcal{C}_{G(4,2,2)} ; q\right)=\left(1+q^{2}\right)^{2}(1+q)^{2}=\sum_{g \in G(4,2,2)} q^{\text {stat }_{\mathrm{G}(4,2,2)}(\mathrm{g})}
$$

from Equation (7), because for each $h \in H$ the partial sum in Equation (8) over the coset $g_{h} N$ is given by

$$
\begin{aligned}
\sum_{n \in N} q^{2 \cdot \operatorname{inv}_{C_{2} \times C_{2}}(1,1)+\operatorname{inv}_{N}(n)} & =q^{0+0}+q^{0+1}+q^{0+1}+q^{0+2} \\
\sum_{n \in N} q^{2 \cdot \operatorname{inv}_{C_{2} \times C_{2}}(-1,1)+\operatorname{inv}_{N}(n)} & =q^{2+0}+q^{2+1}+q^{2+1}+q^{2+2} \\
\sum_{n \in N} q^{2 \cdot \operatorname{inv}_{C_{2} \times C_{2}}(1,-1)+\operatorname{inv}_{N}(n)} & =q^{2+0}+q^{2+1}+q^{2+1}+q^{2+2} \\
\sum_{n \in N} q^{2 \cdot \operatorname{inv}_{C_{2} \times C_{2}}(-1,-1)+\operatorname{inv}_{N}(n)} & =q^{4+0}+q^{4+1}+q^{4+1}+q^{4+2}
\end{aligned}
$$


The factor of 2 in $2 \cdot \operatorname{inv}_{C_{2} \times C_{2}}$ above arises from the grading on the reflection representation $E$ of $H \simeq C_{2} \times C_{2}$ as described in Remark 3.1: in this case we have that $E^{*}=\operatorname{span}_{\mathbb{C}}\left\{x^{2}+y^{2}, x y\right\}$ is homogeneous of degree 2 .

We can similarly obtain a combinatorial interpretation for

$$
\operatorname{Hilb}\left(\mathcal{C}_{G_{9}} ; q\right)=\left(\frac{1-q^{8}}{1-q}\right)\left(\frac{1-q^{24}}{1-q}\right)=\sum_{g \in G_{9}} q^{\text {stat }_{G_{9}}(g)}
$$

from the choice of normal reflection subgroup $N=G(4,2,2)$, which has the combinatorial interpretation $\operatorname{Hilb}\left(\mathcal{C}_{N} ; q\right)=\sum_{n \in N} q^{\text {stat }_{N}(n)}$ obtained in the previous example, and $H \simeq$ $G(6,6,2)$, which is isomorphic as a reflection group to the dihedral group of order 12 and is therefore real and admits the combinatorial interpretation

$$
\operatorname{Hilb}\left(\mathcal{C}_{G(6,6,2)} ; q\right)=\left(\frac{1-q^{2}}{1-q}\right)\left(\frac{1-q^{6}}{1-q}\right)=\sum_{h \in G(6,6,2)} q^{\operatorname{inv}_{G(6,6,2)}(h)} .
$$

Now given a choice of coset representatives $g_{h} \in G_{9}$ for each $h \in H \simeq G(6,6,2)$ we can define, for each $g=g_{h} n \in g_{h} N$,

$$
\operatorname{stat}_{G_{9}}(g)=4 \cdot \operatorname{inv}_{G(6,6,2)}(h)+\operatorname{stat}_{G(4,2,2)}(n),
$$

which is seen to satisfy Equation (9). The factor of 4 in $4 \cdot \operatorname{inv}_{G(6,6,2)}$ above arises from the grading on the reflection representation $E$ of $H \simeq G(6,6,2)$ as described in Remark 3.1: in this case $E^{*}=\operatorname{span}_{\mathbb{C}}\left\{x^{4}+y^{4}, x^{2} y^{2}\right\}$ is homogeneous of degree 4 .

Remark 3.5. It follows from Proposition 3.3 that for any $G$-module $M$ the (dual) OrlikSolomon space $\left(U_{M}^{N}\right)^{*}=\left(\mathcal{C}_{N} \otimes M^{*}\right)^{N}$ as in Definition 2.9 can be considered as an $H$ module or (equivalently) as a $G$-module of $N$-invariants.

The following result is useful in determining the Orlik-Solomon space $U_{M}^{N}$ in particular examples (cf. Section 7) up to graded $G$-module isomorphism.

Lemma 3.6. Let $\eta_{N}: S\left(V^{*}\right) \rightarrow \mathcal{C}_{N}$ denote the G-equivariant projection onto the space of $N$-harmonic polynomials. Let $M$ be a $G$-module of rank $m$, and suppose that $\tilde{u}_{1}, \ldots, \tilde{u}_{m}$ form a homogeneous basis for $\left(S\left(V^{*}\right) \otimes M^{*}\right)^{N}$ as a free $S\left(V^{*}\right)^{N}$-module such that $\left(\tilde{U}_{M}^{N}\right)^{*}:=\operatorname{span}_{\mathbb{C}}\left\{\tilde{u}_{1}, \ldots, \tilde{u}_{m}\right\}$ is G-stable. Then the restriction of the projection $\eta_{N} \otimes 1:$ $\left(\tilde{U}_{M}^{N}\right)^{*} \rightarrow\left(U_{M}^{N}\right)^{*}$ is a graded G-module isomorphism.

Proof. Let $u_{1}, \ldots, u_{m}$ be a homogeneous basis for $\left(U_{M}^{N}\right)^{*}:=\left(\mathcal{C}_{N} \otimes M^{*}\right)^{N}$. We may assume that $\operatorname{deg}\left(u_{i}\right)=e_{i}^{N}(M)=\operatorname{deg}\left(\tilde{u}_{i}\right)$ for each $i=1, \ldots, m$. Let $y_{1}, \ldots, y_{m}$ be a basis for $M^{*}$, and write $\tilde{u}_{i}:=\sum_{j=1}^{m} \tilde{a}_{i j} \otimes y_{j}$ and $u_{i}=\sum_{j=1}^{m} a_{i j} \otimes y_{j}$, where $\tilde{a}_{i j} \in S\left(V^{*}\right)$ and $a_{i j} \in \mathcal{C}_{N}$ and every nonzero $\tilde{a}_{i j}$ and $a_{i j}$ is homogeneous of degree $e_{i}^{N}(M)$. Because the $\tilde{u}_{i}$ and the $u_{i}$ form bases for $\left(S\left(V^{*}\right) \otimes M^{*}\right)^{N}$ as a free $S\left(V^{*}\right)^{N}$-module, there exists a matrix $\left[p_{i j}\right] \in \mathrm{GL}_{m}\left(S\left(V^{*}\right)^{N}\right)$ such that $\left[\tilde{a}_{i j}\right]=\left[p_{i j}\right] \cdot\left[a_{i j}\right]$. Because the kernel of $\eta_{N}: S\left(V^{*}\right) \rightarrow \mathcal{C}_{N}$ is precisely the ideal generated by homogeneous $N$-invariants of positive degree, it follows that $\left[\eta_{N}\left(\tilde{a}_{i j}\right)\right]=\left[p_{i j}(0)\right] \cdot\left[a_{i j}\right]$, where $p_{i j}(0)$ denotes the evaluation at $0 \in V$. Because $\operatorname{deg}\left(\operatorname{det}\left[\tilde{a}_{i j}\right]\right)=\sum_{i=1}^{m} e_{i}^{N}(M)=\operatorname{deg}\left(\operatorname{det}\left[a_{i j}\right]\right)$, it follows that $\operatorname{det}\left[p_{i j}\right] \in \mathbb{C}^{\times}$, and 
therefore $\left[p_{i j}(0)\right] \in \mathrm{GL}_{m}(\mathbb{C})$. Because $\eta_{N} \otimes 1$ is $G$-equivariant and $\left(\tilde{U}_{M}^{N}\right)^{*}$ is $G$-stable, $\eta_{N} \otimes 1:\left(\tilde{U}_{M}^{N}\right)^{*} \rightarrow\left(U_{M}^{N}\right)^{*}$ is a graded isomorphism of $G$-modules.

\subsection{Numerology}

The following consequence of Proposition 3.3 establishes the first equation of Theorem 1.3 in more generality.

Corollary 3.7. Let $M$ be a $G$-module and define $U_{M}^{N}$ as in Definition 2.9. For a suitable choice of indexing we have

$$
e_{i}^{N}(M)+e_{i}^{G}\left(U_{M}^{N}\right)=e_{i}^{G}(M)
$$

Proof. Applying Proposition 3.3, we see that

$$
\left(\mathcal{C}_{G} \otimes M^{*}\right)^{G} \simeq\left(\left(\mathcal{C}_{H} \otimes \mathcal{C}_{N} \otimes M^{*}\right)^{N}\right)^{H}=\left(\mathcal{C}_{H} \otimes\left(\mathcal{C}_{N} \otimes M^{*}\right)^{N}\right)^{H}=\left(\mathcal{C}_{H} \otimes\left(U_{M}^{N}\right)^{*}\right)^{H} .
$$

Let $m$ be the rank of $M$, and let $y_{1}, \ldots, y_{m}$ be a basis of $M^{*}$. Let $a_{i j}^{N} \in \mathcal{C}_{N}$ be $\mathbf{x}$-homogeneous such that $u_{i}^{N}:=\sum_{j=1}^{m} a_{i j}^{N} \otimes y_{j}$ form a basis for $\left(U_{M}^{N}\right)^{*}$ with $\operatorname{deg}_{\mathbf{x}}\left(u_{i}^{N}\right)=e_{i}^{N}(M)$. Letting $\mathcal{E}_{M}^{N}:=\left\{e_{1}^{N}(M), \ldots, e_{m}^{N}(M)\right\}$ denote the set of $M$-exponents of $N$, we see that

$$
\left(U_{M}^{N}\right)^{*} \simeq \bigoplus_{e \in \mathcal{E}_{M}^{N}}\left(U_{M}^{N}\right)_{e}^{*}
$$

as an $H$-module. Therefore, there exist $\mathbf{x}$-homogeneous $a_{i j}^{H} \in \mathcal{C}_{H}$ such that $u_{i}^{G}:=$ $\sum_{j=1}^{m} a_{i j}^{H} \otimes u_{j}^{N}$ form a basis for $\left(\mathcal{C}_{H} \otimes\left(U_{M}^{N}\right)^{*}\right)^{H}$ with $\operatorname{deg}_{\mathbf{x}}\left(u_{i}^{G}\right)=e_{i}^{G}\left(U_{M}^{N}\right)$ and such that $a_{i j}^{H}=0$ whenever $\operatorname{deg}_{\mathbf{x}}\left(u_{j}^{N}\right) \neq e_{i}^{N}(M)$ (in other words, the $\left[a_{i j}^{H}\right]$ may be chosen to be square-diagonal corresponding to the graded decomposition of $\left.\left(U_{M}^{N}\right)^{*}\right)$. But then the $u_{i}^{G}$ form an x-homogeneous basis for $\left(\mathcal{C}_{G} \otimes M^{*}\right)^{G} \simeq\left(\mathcal{C}_{H} \otimes\left(U_{M}^{N}\right)^{*}\right)^{H}$, and we see that

$$
e_{i}^{G}(M)=\operatorname{deg}_{\mathbf{x}}\left(u_{i}^{G}\right)=e_{i}^{G}\left(U_{M}^{N}\right)+e_{i}^{N}(M) .
$$

\subsection{Amenability}

Recall from Section 2.4 that a $G$-module $M$ of rank $m$ is called amenable if $\sum_{i=1}^{m} e_{i}^{G}(M)=$ $e_{1}^{G}\left(\bigwedge^{m} M\right)$.

Remark 3.8. Regardless of whether a $G$-module $M$ of rank $m$ is amenable, we always have a natural $S\left(V^{*}\right)^{G}$-linear injective homomorphism

$$
S\left(V^{*}\right)^{G} \otimes \bigwedge^{m}\left(\mathcal{C}_{G} \otimes M^{*}\right)^{G} \hookrightarrow S\left(V^{*}\right)^{G} \otimes\left(\mathcal{C}_{G} \otimes \bigwedge^{m} M^{*}\right)^{G}
$$

in $\left(S\left(V^{*}\right) \otimes \bigwedge M^{*}\right)^{G}$, which identifies $\bigwedge^{m}\left(\mathcal{C}_{G} \otimes M^{*}\right)^{G}$ with $a \otimes\left(\mathcal{C}_{G} \otimes \bigwedge^{m} M^{*}\right)^{G}$ for some $0 \neq a \in S\left(V^{*}\right)^{G}$. The amenability of $M$ as a $G$-module is therefore precisely the requirement that $a \in \mathbb{C}$ be a constant polynomial. For brevity and convenience, we will summarise this equivalent characterisation of amenability in the following Lemma 3.9 (cf. [7, Theorem 2.10]).

Lemma 3.9. A G-module $M$ of rank $m$ is amenable if and only if

$$
\bigwedge^{m}\left(\left(\mathcal{C}_{G} \otimes M^{*}\right)^{G}\right) \simeq\left(\mathcal{C}_{G} \otimes \bigwedge^{m} M^{*}\right)^{G} .
$$


The decomposition $\mathcal{C}_{G} \simeq \mathcal{C}_{H} \otimes \mathcal{C}_{N}$ from Proposition 3.3 and its Corollary 3.7 have many useful consequences. Although the following result can be proved more directly by appealing to [7, Corollary 8.7], we provide a full proof.

Lemma 3.10. Let $M$ be an H-module. Then

(i) $\left(\mathcal{C}_{G} \otimes M^{*}\right)^{G} \simeq\left(\mathcal{C}_{H} \otimes M^{*}\right)^{H}$ and

(ii) $M$ is amenable as a $G$-module if and only if $M$ is amenable as an $H$-module.

Proof. The $M$-exponents $e_{i}^{G}(M)$ of $G$ are obtained as the x-degrees of any $\mathbf{x}$ homogeneous basis for $\left(\mathcal{C}_{G} \otimes M^{*}\right)$, and the $M$-exponents $e_{i}^{H}(M)$ of $H$ are analogously obtained as the $\mathbf{N}$-degrees of any (bi)homogeneous basis for $\left(\mathcal{C}_{H} \otimes M^{*}\right)^{H}$. Letting $m$ denote the rank of $M$, the exponent $e_{1}^{G}\left(\bigwedge^{m} M\right)$ is the $\mathbf{x}$-degree of a basis element for $\left(\mathcal{C}_{G} \otimes \bigwedge^{m} M^{*}\right)^{G}$, and the exponent $e_{i}^{H}\left(\bigwedge^{m} M\right)$ is the $\mathbf{N}$-degree of a basis element for $\left(\mathcal{C}_{H} \otimes \bigwedge^{m} M^{*}\right)^{H}$. Because both $M$ and $\mathcal{C}_{H}$ are $N$-invariant, and $\mathcal{C}_{G} \simeq \mathcal{C}_{H} \otimes \mathcal{C}_{N}$ as graded $G$-modules such that $\left(\mathcal{C}_{G}\right)^{N} \simeq \mathcal{C}_{H}$ by Proposition 3.3, we obtain

$$
\left(\mathcal{C}_{G} \otimes M^{*}\right)^{G}=\left(\left(\mathcal{C}_{G} \otimes M^{*}\right)^{N}\right)^{H}=\left(\left(\mathcal{C}_{G}\right)^{N} \otimes M^{*}\right)^{H} \simeq\left(\mathcal{C}_{H} \otimes M^{*}\right)^{H},
$$

which establishes (i).

Applying (i) to $\bigwedge^{m} M^{*}$, we have that $\left(\mathcal{C}_{G} \otimes \bigwedge^{m} M^{*}\right)^{G} \simeq\left(\mathcal{C}_{H} \otimes \bigwedge^{m} M^{*}\right)^{H}$. By Lemma 3.9, the amenability of $M$ as a $G$-module and the amenability of $M$ as an $H$-module are respectively equivalent to

$$
\bigwedge^{m}\left(\left(\mathcal{C}_{G} \otimes M^{*}\right)^{G}\right) \simeq\left(\mathcal{C}_{G} \otimes \bigwedge^{m} M^{*}\right)^{G} \quad \text { and } \quad \bigwedge^{m}\left(\left(\mathcal{C}_{H} \otimes M^{*}\right)^{H}\right) \simeq\left(\mathcal{C}_{H} \otimes \bigwedge^{m} M^{*}\right)^{H},
$$

which proves (ii).

Remark 3.11. Because the Orlik-Solomon space $U_{M}^{N}$ of Definition 2.9 is trivial as an $N$-module, it follows from Lemma 3.10 that $U_{M}^{N}$ is amenable as a $G$-module if and only if it is amenable as an $H$-module. From now on we will just say that $U_{M}^{N}$ is amenable whenever these equivalent conditions hold.

Proposition 3.12. Let $M$ be a G-module and define $U_{M}^{N}$ as in Definition 2.9.

(i) If $M$ is amenable as a $G$-module, then $U_{M}^{N}$ is amenable.

(ii) If $M$ is amenable as an $N$-module and $U_{M}^{N}$ is amenable, then $M$ is amenable as a G-module.

Proof. Let $m$ denote the rank of $M$. Define the amenability defects

$$
\begin{gathered}
\gamma:=\sum_{i=1}^{m} e_{i}^{G}(M)-e_{1}^{G}\left(\bigwedge^{m} M\right) ; \quad \nu:=\sum_{i=1}^{m} e_{i}^{N}(M)-e_{1}^{N}\left(\bigwedge^{m} M\right) ; \quad \text { and } \\
\eta:=\sum_{i=1}^{m} e_{i}^{G}\left(U_{M}^{N}\right)-e_{1}^{G}\left(\bigwedge^{m} U_{M}^{N}\right),
\end{gathered}
$$

so that $M$ is amenable as a $G$-module if and only if $\gamma=0, M$ is amenable as an $N$-module if and only if $\nu=0$ and $U_{M}^{N}$ is amenable as a $G$-module if and only if $\eta=0$. By Lemma 3.10, 
$U_{M}^{N}$ is also amenable as an $H$-module if and only if $\eta=0$. By [16, Lemma 2.8] (see also Remark 3.8), in any case we have that $\gamma, \nu, \eta \geq 0$.

Let $W_{M}^{N}$ be the dual of $\left(W_{M}^{N}\right)^{*}:=\left(\mathcal{C}_{N} \otimes \bigwedge^{m} M^{*}\right)^{N}$ (instead of the more natural but cumbersome $U_{\wedge^{m} M}^{N}$ in lieu of $\left.W_{M}^{N}\right)$. From Corollary 3.7, we know that

$$
e_{i}^{N}(M)+e_{i}^{G}\left(U_{M}^{N}\right)=e_{i}^{G}(M) \quad \text { and } \quad e_{1}^{N}\left(\bigwedge^{m} M\right)+e_{1}^{G}\left(W_{M}^{N}\right)=e_{1}^{G}\left(\bigwedge^{m} M\right) .
$$

Summing the first equation over $i=1, \ldots, m$ and subtracting the second equation, we obtain

$$
\nu+\eta+e_{1}^{G}\left(\bigwedge^{m} U_{M}^{N}\right)=\gamma+e_{1}^{G}\left(W_{M}^{N}\right) .
$$

Consider now the natural inclusion of graded $G$-modules

$$
S\left(E^{*}\right) \otimes \bigwedge^{m}\left(\mathcal{C}_{N} \otimes M^{*}\right)^{N} \hookrightarrow S\left(E^{*}\right) \otimes\left(\mathcal{C}_{N} \otimes \bigwedge^{m} M^{*}\right)^{N}
$$

in $\left(S\left(V^{*}\right) \otimes \bigwedge M^{*}\right)^{N}$, which identifies $\bigwedge^{m}\left(U_{M}^{N}\right)^{*}$ with $a \otimes\left(W_{M}^{N}\right)^{*}$ as graded $G$-modules for some $\mathbf{x}$-homogeneous $a \in S\left(E^{*}\right)$ with $\operatorname{deg}_{\mathbf{x}}(a)=\nu$ (cf. Remark 3.8). From this it follows that $e_{1}^{G}\left(\bigwedge^{m} U_{M}^{N}\right)+\nu \geq e_{1}^{G}\left(W_{M}^{N}\right)$, which together with Equation (10) implies that $\eta \leq \gamma$. Therefore, if $M$ is amenable as a $G$-module, then $U_{M}^{N}$ is amenable. If $M$ is amenable as an $N$-module, so that $\nu=0$, then we see that $\bigwedge^{m}\left(U_{M}^{N}\right)^{*} \simeq\left(W_{M}^{N}\right)^{*}$ as $G$-modules. Therefore, $e_{1}^{G}\left(\bigwedge^{m} U_{M}^{N}\right)=e_{1}^{G}\left(W_{M}^{N}\right)$, and we obtain from Equation (10) that $\eta=\gamma$ in this case. Hence, if $M$ is amenable as an $N$-module and $U_{M}^{N}$ is amenable, then $M$ is amenable as a $G$-module.

Remark 3.13. It is not true in general that $M$ being amenable as a $G$-module implies that $M$ is amenable as an $N$-module. For a counterexample, let $G=C_{a}=\langle c\rangle$ and $N=$ $C_{d}=\left\langle c^{e}\right\rangle$ with $a=d e$, acting on $V=\mathbb{C}$ in the standard reflection representation by $c \mapsto \zeta_{a}$, a primitive $a$ th root of unity. Consider the $G$-module $M:=V^{*} \oplus\left(V^{*}\right)^{\otimes(d-1)}$, so that $\bigwedge^{2} M \simeq\left(V^{*}\right)^{\otimes d}$. Then $e_{1}^{G}(M)=1=e_{1}^{N}(M)$ and $e_{2}^{G}(M)=d-1=e_{2}^{N}(M)$. Because $e_{1}^{G}\left(\bigwedge^{2} M\right)=d=e_{1}^{G}(M)+e_{2}^{G}(M), M$ is amenable as a $G$-module. However, $\bigwedge^{2} M$ is trivial as an $N$-module, and therefore $e_{1}^{N}\left(\bigwedge^{2} M\right)=0 \neq d=e_{1}^{N}(M)+e_{2}^{N}(M)$, so $M$ is not amenable as an $N$-module.

\subsection{Poincaré Series}

Suppose that $M$ is an amenable $G$-module of rank $m$. Then by Proposition 3.12 and Lemma 3.10, the Orlik-Solomon space $U_{M}^{N}$ of Definition 2.9 is amenable (considered either as a $G$-module or as an $H$-module). Let us again write $\mathcal{E}_{M}^{N}:=\left\{e_{1}^{N}(M), \ldots, e_{m}(M)\right\}$ for the set of $M$-exponents of $N$. We have a graded $G$-module decomposition

$$
\left(U_{M}^{N}\right)^{*}=\bigoplus_{e \in \mathcal{E}_{M}^{N}}\left(U_{M}^{N}\right)_{e}^{*},
$$

from which we obtain more generally a graded $G$-module decomposition of $\bigwedge^{p}\left(U_{M}^{N}\right)^{*}$ :

$$
\left(\bigwedge^{p}\left(U_{M}^{N}\right)^{*}\right)_{e}:=\operatorname{span}_{\mathbb{C}}\left\{u_{i_{1}} \wedge \cdots \wedge u_{i_{p}} \mid u_{i_{j}} \in\left(U_{M}^{N}\right)_{e_{j}}^{*} \text { with } \sum_{j=1}^{p} e_{j}=e\right\} .
$$


This results in an obvious bigrading of $\bigwedge\left(U_{M}^{N}\right)^{*}$ and a corresponding trigrading of the associative algebra $S\left(V^{*}\right) \otimes \bigwedge\left(U_{M}^{N}\right)^{*}$.

Definition 3.14. The trigraded Poincaré series for $\left(S\left(V^{*}\right) \otimes \wedge\left(U_{M}^{N}\right)^{*}\right)^{G}$ is

$$
\mathcal{P}_{M}^{G}(x, y, u):=\sum_{\ell, e, p \geq 0} \operatorname{dim}_{\mathbb{C}}\left(S\left(V^{*}\right)_{\ell} \otimes\left(\bigwedge^{p}\left(U_{M}^{N}\right)^{*}\right)_{e}\right)^{G} x^{\ell} y^{e} u^{p} .
$$

We will follow the usual strategy of computing this Poincaré series in two different ways to deduce combinatorial formulas. Let us denote as before $d_{1}^{G}, \ldots, d_{r}^{G}$ the degrees of the fundamental $G$-invariants generating $S\left(V^{*}\right)^{G}$ as a polynomial algebra. Let us index the $M$-exponents of $N, e_{1}^{N}(M), \ldots, e_{m}^{N}(M)$, and the $U_{M}^{N}$-exponents of $G, e_{1}^{G}\left(U_{M}^{N}\right), \ldots, e_{m}^{G}\left(U_{M}^{N}\right)$, as in Corollary 3.7, so that $e_{i}^{N}(M)+e_{i}^{G}\left(U_{M}^{N}\right)=e_{i}^{G}(M)$.

Proposition 3.15. $\mathcal{P}_{M}^{G}(x, y, u)=\frac{\prod_{i=1}^{m}\left(1+x^{e_{i}^{G}\left(U_{M}^{N}\right)} y^{e_{i}^{N}(M)} u\right)}{\prod_{j=1}^{r}\left(1-x^{d_{j}^{G}}\right)}$.

Proof. We proceed as in the proof of Corollary 3.7: let $y_{1}, \ldots, y_{m}$ be a basis of $M^{*}$. Let $a_{i j}^{N} \in \mathcal{C}_{N}$ be $\mathrm{x}$-homogeneous such that $u_{i}^{N}:=\sum_{j=1}^{m} a_{i j}^{N} \otimes y_{j}$ form a basis for the $e_{i}^{N}(M)$ homogeneous component of $\left(U_{M}^{N}\right)^{*}$, and choose $\mathbf{x}$-homogeneous $a_{i j}^{H} \in \mathcal{C}_{H}$ such that $u_{i}^{G}:=$ $\sum_{j=1}^{m} a_{i j}^{H} \otimes u_{j}^{N}$ form a basis for $\left(\mathcal{C}_{H} \otimes\left(U_{M}^{N}\right)_{e_{i}^{N}(M)}^{*}\right)^{H}$ with $\operatorname{deg}_{\mathbf{x}}\left(u_{i}^{G}\right)=e_{i}^{G}\left(U_{M}^{N}\right)$ (where again $a_{i j}^{H}=0$ whenever $\left.\operatorname{deg}_{\mathbf{x}}\left(u_{j}^{N}\right) \neq e_{i}^{N}(M)\right)$. But then the $u_{i}^{G}$ form an $\mathbf{x}$-homogeneous basis for $\left(\mathcal{C}_{H} \otimes\left(U_{M}^{N}\right)^{*}\right)^{H}$. Because $U_{M}^{N}$ consists of $N$-invariants, by Lemma 3.10 we have that $\left(\mathcal{C}_{G} \otimes\left(U_{M}^{N}\right)^{*}\right)^{G} \simeq\left(\mathcal{C}_{H} \otimes\left(U_{M}^{N}\right)^{*}\right)^{H}$. By Proposition 3.12, because $M$ is amenable as a $G$-module, $U_{M}^{N}$ is amenable. Hence, by Theorem 2.7 we have that

$$
\left(S\left(V^{*}\right) \otimes \bigwedge\left(U_{M}^{N}\right)^{*}\right)^{G} \simeq S\left(V^{*}\right)^{G} \otimes \bigwedge\left(\mathcal{C}_{G} \otimes\left(U_{M}^{N}\right)^{*}\right)^{G} .
$$

Because $\left(\mathcal{C}_{G} \otimes\left(U_{M}^{N}\right)^{*}\right)^{G} \simeq \operatorname{span}_{\mathbb{C}}\left\{u_{1}^{G}, \ldots, u_{m}^{G}\right\}$ and $u_{i}^{G} \in S\left(V^{*}\right)_{e_{i}^{G}\left(U_{M}^{N}\right)} \otimes\left(U_{M}^{N}\right)_{e_{i}^{N}(M)}^{*}$, our result follows.

To simplify notation, for $e \in \mathcal{E}_{M}^{N}$ we denote by $\left(U_{M}^{N}\right)_{e}$ the homogeneous component of $U_{M}^{N}$ corresponding to the dual of $\left(U_{M}^{N}\right)_{e}^{*}$, rather than the more natural but cumbersome graded dual $\left(U_{M}^{N}\right)_{-e}$ instead of our $\left(U_{M}^{N}\right)_{e}$.

Proposition 3.16. $\mathcal{P}_{M}^{G}(x, y, u)=\frac{1}{|G|} \sum_{g \in G} \frac{\prod_{e \in \mathcal{E}_{M}^{N}} \operatorname{det}\left(1+y^{e} u g \mid\left(U_{M}^{N}\right)_{e}\right)}{\operatorname{det}(1-x g \mid V)}$.

Proof. For $g \in G$, let us write

$$
\mathcal{P}_{M}^{g}(x, y, u)=\sum_{\ell, e, p \geq 0} \operatorname{tr}\left(g \mid S\left(V^{*}\right)_{\ell} \otimes\left(\bigwedge^{p}\left(U_{M}^{N}\right)^{*}\right)_{e}\right) x^{\ell} y^{e} u^{p},
$$

so that $\mathcal{P}_{M}^{G}(x, y, u)=\frac{1}{|G|} \sum_{g \in G} \mathcal{P}_{M}^{g}(x, y, u)$ and

$$
\mathcal{P}_{M}^{g}(x, y, u)=\left(\sum_{\ell \geq 0} \operatorname{tr}\left(g \mid S\left(V^{*}\right)_{\ell}\right) x^{\ell}\right)\left(\sum_{e, p \geq 0} \operatorname{tr}\left(g \mid\left(\bigwedge^{p}\left(U_{M}^{N}\right)^{*}\right)_{e}\right) y^{e} u^{p}\right) .
$$


We know that $\sum_{\ell \geq 0} \operatorname{tr}\left(g \mid S\left(V^{*}\right)_{\ell}\right) x^{\ell}=\operatorname{det}\left(1-x g^{-1} \mid V\right)^{-1}$. On the other hand, because $\left(U_{M}^{N}\right)^{*} \simeq \bigoplus_{e \in \mathcal{E}_{M}^{N}}\left(U_{M}^{N}\right)_{e}^{*}$, we have that $\bigwedge\left(U_{M}^{N}\right)^{*} \simeq \bigotimes_{e \in \mathcal{E}_{M}^{N}} \wedge\left(U_{M}^{N}\right)_{e}^{*}$ as bigraded $G$-modules. Hence, for each $g \in G$,

$$
\sum_{e, p \geq 0} \operatorname{tr}\left(g \mid\left(\bigwedge^{p}\left(U_{M}^{N}\right)^{*}\right)_{e}\right) y^{e} u^{p}=\prod_{e \in \mathcal{E}_{M}^{N}}\left(\sum_{p \geq 0} \operatorname{tr}\left(g \mid \bigwedge^{p}\left(U_{M}^{N}\right)_{e}^{*}\right) y^{e p} u^{p}\right) .
$$

For each $e \in \mathcal{E}_{M}^{N}$ we have that $\sum_{p \geq 0} \operatorname{tr}\left(g \mid \bigwedge^{p}\left(U_{M}^{N}\right)_{e}^{*}\right) y^{e p} u^{p}=\operatorname{det}\left(1+y^{e} u g^{-1} \mid\left(U_{M}^{N}\right)_{e}\right)$. Hence, for each $g \in G$,

$$
\mathcal{P}_{M}^{g}(x, y, u)=\frac{\prod_{e \in \mathcal{E}_{M}^{N}} \operatorname{det}\left(1+y^{e} u g^{-1} \mid\left(U_{M}^{N}\right)_{e}\right)}{\operatorname{det}\left(1-x g^{-1} \mid V\right)} .
$$

Our result follows after taking the average over $g \in G$ on each side.

\section{Proofs of the Main Theorems}

We are now in a position to apply the results of Section 3 to prove the main results announced in the Introduction. Fix $G \subset \mathrm{GL}(V)$ a complex reflection group acting by reflections on the vector space $V$ of dimension $r$. Let $N \unlhd G$ be a normal reflection subgroup with quotient $H=G / N$, which acts by reflections on $E=V / N$. For $\sigma \in$ $\operatorname{Gal}\left(\mathbb{Q}\left(\zeta_{G}\right) / \mathbb{Q}\right)$, where $\zeta_{G}$ denotes a primitive $|G|$ th root of unity, write $V^{\sigma}$ for the Galois twist of $V$ (as defined in Section 2.3). As in Definition 2.9, we write $U^{N}$ for the dual of $\left(\mathcal{C}_{N} \otimes V^{*}\right)^{N}$ and, more generally, $U_{\sigma}^{N}$ for the dual of $\left(\mathcal{C}_{N} \otimes\left(V^{\sigma}\right)^{*}\right)^{N}$.

\subsection{Proof of Theorem 1.3}

Theorem 1.3. Let $G \subset \mathrm{GL}(V)$ be a complex reflection group and let $N \unlhd G$ be a normal reflection subgroup. Let $H=G / N$ and $E=V / N$. Then for a suitable choice of indexing we have

$$
\begin{aligned}
e_{i}^{N}\left(V^{\sigma}\right)+e_{i}^{G}\left(U_{\sigma}^{N}\right) & =e_{i}^{G}\left(V^{\sigma}\right) \\
d_{i}^{N} \cdot e_{i}^{H}\left(E^{\sigma}\right) & =e_{i}^{G}\left(E^{\sigma}\right) \\
d_{i}^{N} \cdot d_{i}^{H} & =d_{i}^{G} .
\end{aligned}
$$

Proof. The first equality is Corollary 3.7 applied to the $G$-module $M=V^{\sigma}$, and the last equality follows from the observations in Remark 3.1.

Let us establish the second equality. Let $N_{1}^{\sigma}, \ldots, N_{r}^{\sigma}$ denote a basis for $\left(E^{\sigma}\right)^{*}$ as a $G$ module. We will show that there exist $a_{i j} \in S\left(E^{*}\right)$ such that $u_{i}:=\sum_{j=1}^{r} a_{i j} \otimes N_{j}^{\sigma}$ form an $\mathbf{N}$-homogeneous basis for $\left(\mathcal{C}_{H} \otimes\left(E^{\sigma}\right)^{*}\right)^{H}$ (so that each nonzero $a_{i j}$ is $\mathbf{N}$-homogeneous of $\mathbf{N}$-degree $e_{i}^{H}\left(E^{\sigma}\right)$ ) and, moreover, $a_{i j}=0$ whenever $d_{j}^{N} \neq d_{i}^{N}$ and each $a_{i j} \in S\left(\mathbf{N}_{d_{i}^{N}}\right)$, where as before $\mathbf{N}_{d}$ denotes the set of fundamental $N$-invariants of degree $d$. Because $\left(\mathcal{C}_{G} \otimes\left(E^{\sigma}\right)^{*}\right)^{G} \simeq\left(\mathcal{C}_{H} \otimes\left(E^{\sigma}\right)^{*}\right)^{H}$ by Lemma 3.10 , the existence of such $a_{i j}$ will establish our claim, because each nonzero $a_{i j}$ as above will then be $\mathbf{x}$-homogeneous of $\mathbf{x}$-degree $e_{i}^{G}\left(E^{\sigma}\right)=d_{i}^{N} \cdot e_{i}^{H}\left(E^{\sigma}\right)$. 
As in Remark 3.1, let us write $\mathcal{D}_{N}=\left\{d_{1}^{N}, \ldots, d_{r}^{N}\right\}$ for the set of degrees of $N$ and $E_{d}^{*}$ for the graded component of $E^{*}$ spanned by the fundamental $N$-invariants of degree $d \in \mathcal{D}_{N}$. To simplify notation, let us write $E_{d}$ for the graded dual of $E_{d}^{*}$, instead of $E_{-d}$, so that $E \simeq \bigoplus_{d \in \mathcal{D}_{N}} E_{d}$, and similarly the Galois twist $E^{\sigma} \simeq \bigoplus_{d \in \mathcal{D}_{N}} E_{d}^{\sigma}$. We saw in Remark 3.1 that $H$ decomposes as a direct product $\chi_{d \in \mathcal{D}_{N}} H_{(d)}$, where each $H_{(d)}$ is a reflection group acting on $E_{d}$ and $H_{(d)}$ acts trivially on $E_{d^{\prime}}^{\sigma}$ whenever $d \neq d^{\prime}$. We then see that $S\left(E^{*}\right) \simeq \bigotimes_{d \in \mathcal{D}_{N}} S\left(E_{d}^{*}\right)$ and each $S\left(E_{d}^{*}\right) \simeq S\left(E_{d}^{*}\right)^{H_{(d)}} \otimes \mathcal{C}_{H_{(d)}}$ as $H$-modules, so that in particular $\mathcal{C}_{H} \simeq \bigotimes_{d \in \mathcal{D}_{N}} \mathcal{C}_{H_{(d)}}$ as $H$-modules, where again $H_{(d)}$ acts trivially on $\mathcal{C}_{H_{\left(d^{\prime}\right)}}$ for $d \neq d^{\prime}$. It follows from the above observations that

$$
\left(\mathcal{C}_{H} \otimes\left(E^{\sigma}\right)^{*}\right)^{H} \simeq\left(\bigotimes_{d \in \mathcal{D}_{N}} \mathcal{C}_{H_{(d)}} \otimes \bigoplus_{d \in \mathcal{D}_{N}}\left(E_{d}^{\sigma}\right)^{*}\right)^{\times_{d \in \mathcal{D}_{N}} H_{(d)}} \simeq \bigoplus_{d \in \mathcal{D}_{N}}\left(\mathcal{C}_{H_{(d)}} \otimes\left(E_{d}^{\sigma}\right)^{*}\right)^{H_{(d)}},
$$

so that we may indeed choose $a_{i j} \in \mathcal{C}_{H_{\left(d_{i}^{N}\right)}} \subset S\left(E_{d_{i}^{N}}^{*}\right)$ such that the $\mathbf{N}$-homogeneous $u_{i}=$ $\sum_{j=1}^{r} a_{i j} \otimes N_{j}^{\sigma} \in\left(\mathcal{C}_{H_{\left(d_{i}^{N}\right)}} \otimes\left(E_{d_{i}^{N}}^{\sigma}\right)^{*}\right)^{H_{\left(d_{i}^{N}\right)}}$ (i.e., with $a_{i j}=0$ whenever $d_{i}^{N} \neq d_{j}^{N}$ ) form a basis for $\left(\mathcal{C}_{H} \otimes\left(E^{\sigma}\right)^{*}\right)^{H}$ that is simultaneously $\mathbf{N}$-homogeneous of $\mathbf{N}$-degree $e_{i}^{H}\left(E^{\sigma}\right)$ and x-homogeneous of x-degree $e_{i}^{G}\left(E^{\sigma}\right)=d_{i}^{N} \cdot e_{i}^{H}\left(E^{\sigma}\right)$.

When $\sigma=1$, the $G$-module $U_{\sigma}^{N}$ in Definition 2.9 admits a more concrete description.

Lemma 4.1 ([7, Example 2.4]). Let $\eta: S\left(V^{*}\right) \rightarrow \mathcal{C}_{N}$ denote the projection onto the space of $N$-harmonic polynomials, and let

$$
\begin{array}{r}
d: E^{*}=\operatorname{span}_{\mathbb{C}}\left\{N_{1}, \ldots, N_{r}\right\} \rightarrow \operatorname{span}_{\mathbb{C}}\left\{d N_{1}, \ldots, d N_{r}\right\} \\
N_{i} \mapsto d N_{i}=\sum_{j=1}^{r} \frac{\partial N_{i}}{\partial x_{j}} \otimes x_{j} .
\end{array}
$$

Then $(\eta \otimes 1) \circ d: E^{*} \rightarrow\left(U^{N}\right)^{*}$ is a graded (of degree -1$)$ isomorphism of G-modules.

Remark 4.2. As mentioned in the Introduction, in the case where $\sigma=1$, once we replace $e_{i}^{G}\left(U^{N}\right)=e_{i}^{G}(E)$ by Lemma 4.1, the equalities in Theorem 1.3 are compatible with the classical relations $d_{i}^{G}=e_{i}^{G}(V)+1, d_{i}^{N}=e_{i}^{N}(V)+1$ and $d_{i}^{H}=e_{i}^{H}(E)+1$. To see this, we proceed as in [1, Theorem 1.3]. We found in Remark 3.1 a choice of $\mathbf{N}$-homogeneous $H$-invariants $H_{i}\left(\mathbf{N}_{d_{i}^{N}}\right)=G_{i}(\mathbf{x})$, a set of fundamental $G$-invariants as in Equation (5), immediately resulting in the equality $d_{i}^{N} \cdot d_{i}^{H}=d_{i}^{G}$ of Theorem 1.3. Let us show that this same choice of indexing results in the other two equalities of Theorem 1.3. We begin by comparing $\mathbf{x}$-degrees in

$$
d G_{i}=\sum_{j=1}^{r} \frac{\partial G_{i}}{\partial x_{j}} \otimes x_{j}=\sum_{k=1}^{r} \frac{\partial H_{i}}{\partial N_{k}} \cdot d N_{k}=\sum_{k=1}^{r} \sum_{j=1}^{r} \frac{\partial H_{i}}{\partial N_{k}} \cdot \frac{\partial N_{k}}{\partial x_{j}} \otimes x_{j}
$$

Recall that $e_{i}^{G}(V)=d_{i}^{G}-1=\operatorname{deg}_{\mathbf{x}}\left(d G_{i}\right)$ and $e_{i}^{N}(V)=d_{i}^{N}-1=\operatorname{deg}_{\mathbf{x}}\left(d N_{i}\right)$. Similarly, $e_{i}^{H}(E)=d_{i}^{H}-1=\operatorname{deg}_{\mathbf{N}}\left(d H_{i}\right)$, where now $d H_{i}=\sum_{k=1}^{r} \frac{\partial H_{i}}{\partial N_{k}} \otimes N_{k} \in\left(S\left(E^{*}\right) \otimes E^{*}\right)^{H}$. Because $\frac{\partial H_{i}}{\partial N_{k}}=0$ whenever $\operatorname{deg}_{\mathbf{x}}\left(N_{k}\right) \neq d_{i}^{N}$, it follows that

$$
e_{i}^{G}(V)=e_{i}^{N}(V)+d_{i}^{N} \cdot\left(d_{i}^{H}-1\right)=e_{i}^{N}(V)+d_{i}^{N} \cdot e_{i}^{H}(E) .
$$


It remains to show that $d_{i}^{N} \cdot e_{i}^{H}(E)=e_{i}^{G}(E)$ under this same choice of indexing.

The $e_{i}^{G}(E)$ are the $\mathbf{x}$-degrees of a homogeneous basis for $\left(\mathcal{C}_{G} \otimes E^{*}\right)^{G}$. By Lemma 3.10, $\left(\mathcal{C}_{G} \otimes E^{*}\right)^{G} \simeq\left(\mathcal{C}_{H} \otimes E^{*}\right)^{H}$, and therefore the $\left(\eta_{H} \otimes 1\right)\left(d H_{i}\right)$ serve as a homogeneous basis for $\left(\mathcal{C}_{G} \otimes E^{*}\right)^{G}$, where $\eta_{H}: S\left(E^{*}\right) \rightarrow \mathcal{C}_{H}$ denotes the projection onto the space of $H$ harmonic polynomials (cf. Lemma 4.1). Hence, for any $k$ such that $\frac{\partial H_{i}}{\partial N_{k}} \neq 0$, the $e_{i}^{G}(E)$ are given by $\operatorname{deg}_{\mathbf{x}}\left(\frac{\partial H_{i}}{\partial N_{k}}\right)=\left(d_{i}^{H}-1\right) d_{i}^{N}$.

The proof of the second equality of Theorem 1.3 generalises what is essential in the case $\sigma=1$ - where we have explicit bases for the relevant Orlik-Solomon spaces of Definition 2.9 in terms of fundamental invariants (up to a harmless isomorphism as in Lemma 4.1): $\left\{d G_{i}\right\}$ for $\left(U^{G}\right)^{*},\left\{d N_{i}\right\}$ for $\left(U^{N}\right)^{*}$ and $\left\{d H_{i}\right\}$ for $\left(U^{H}\right)^{*}$ - to the more general situation where $\sigma \in \operatorname{Gal}\left(\mathbb{Q}\left(\zeta_{G}\right) / \mathbb{Q}\right)$ is arbitrary.

Example 4.3. Take $G=W\left(F_{4}\right)=G_{28}, N$ to be the normal subgroup generated by the reflections corresponding to short roots (see the proof of Theorem 6.2 for more details), and $\sigma=1$. Then $N \simeq W\left(D_{4}\right)$ and $G / N \simeq W\left(A_{2}\right)=\mathfrak{S}_{3}$ acts by reflections on $\mathbb{C} \oplus \mathbb{C} \oplus \mathbb{C}^{2}$ (trivially on $\mathbb{C} \oplus \mathbb{C}$ ). Theorem 1.3 corresponds to the identities

$$
\begin{aligned}
& (1,5,3,3)+(0,0,4,8)=(1,5,7,11) \\
& (2,6,4,4) \cdot(0,0,1,2)=(0,0,4,8) \\
& (2,6,4,4) \cdot(1,1,2,3)=(2,6,8,12) .
\end{aligned}
$$

Note that the exponents and degrees of $N \simeq W\left(D_{4}\right)$ must be reordered for the identities to hold (see Remark 3.1).

Remark 4.4. When $\sigma=1, U_{\sigma}^{N} \simeq E^{\sigma}$ as $G$-representations by Lemma 4.1 - but it is not always the case that $U_{\sigma}^{N} \simeq E^{\sigma}$ as $G$-representations for more general $\sigma$. For example, take the cyclic groups $G=C_{a} \triangleright C_{d}=N$ for $d \mid a$, with $\sigma$ being complex conjugation. Then $\left(U_{\sigma}^{N}\right)^{*}=\operatorname{span}_{\mathbb{C}}\left\{x \otimes x^{\sigma}\right\}$, on which $G$ acts trivially. We discuss this in more detail in Section 7.1. See Section 7 for more examples of explicit identifications of the spaces $U_{\sigma}^{N}$.

\subsection{Proof of Theorem 1.4}

Theorem 1.4. Let $G \subset \mathrm{GL}(V)$ be a complex reflection group of rank $r$ and let $N \unlhd G$ be a normal reflection subgroup. Let $E=V / N$ and $\sigma \in \operatorname{Gal}\left(\mathbb{Q}\left(\zeta_{G}\right) / \mathbb{Q}\right)$. Then for a suitable choice of indexing we have

$$
\sum_{g \in G}\left(\prod_{\lambda_{i}(g) \neq 1} \frac{1-\lambda_{i}(g)^{\sigma}}{1-\lambda_{i}(g)}\right) q^{\mathrm{fix}_{V}(g)} t^{\mathrm{fix}_{E}(g)}=\prod_{i=1}^{r}\left(q t+e_{i}^{N}\left(V^{\sigma}\right) t+e_{i}^{G}\left(U_{\sigma}^{N}\right)\right),
$$

where the $\lambda_{i}(g)$ are the eigenvalues of $g \in G$ acting on $V$.

We refer to the left-hand side of Theorem 1.4 as the sum side and to the right-hand side as the product side. We will prove Theorem 1.4 by computing the limit as $x \rightarrow 1$ of the specialisation $y \mapsto x^{t}$ and $u \mapsto q t(1-x)-1$ of the trigraded Poincaré series $\mathcal{P}_{\sigma}^{G}(x, y, u):=$ $\mathcal{P}_{V^{\sigma}}^{G}(x, y, u)$ from Definition 3.14 in two different ways to obtain the sum side and the product side separately. 
Proof of Theorem 1.4. By Corollaries 4.5 and 4.11, both sides are equal to $\lim _{x \rightarrow 1}|G|$. $\mathcal{P}_{\sigma}^{G}\left(x, x^{t}, q t(1-x)-1\right)$.

Because $M=V^{\sigma}$ is amenable as a $G$-module (and as an $N$-module) by [16, Thm. 2.13], we can apply both Propositions 3.15 and 3.16 in this case to obtain

$$
\frac{1}{|G|} \sum_{g \in G} \frac{\prod_{e \in \mathcal{E}_{\sigma}^{N}} \operatorname{det}\left(1+y^{e} u g \mid\left(U_{\sigma}^{N}\right)_{e}\right)}{\operatorname{det}(1-x g \mid V)}=\mathcal{P}_{\sigma}^{G}(x, y, u)=\prod_{i=1}^{r} \frac{\left(1+x^{e_{i}^{G}\left(U_{\sigma}^{N}\right)} y^{e_{i}^{N}\left(V^{\sigma}\right)} u\right)}{\left(1-x^{d_{i}^{G}}\right)}
$$

where $\mathcal{E}_{\sigma}^{N}:=\left\{e_{1}^{N}\left(V^{\sigma}\right), \ldots, e_{r}^{N}\left(V^{\sigma}\right)\right\}$ denotes the set of $V^{\sigma}$-exponents of $N$ as before. The product side of Theorem 1.4 follows immediately.

Corollary 4.5 (Product side specialisation).

$$
\lim _{x \rightarrow 1}|G| \cdot \mathcal{P}_{\sigma}^{G}\left(x, x^{t}, q t(1-x)-1\right)=\prod_{i=1}^{r}\left(q t+e_{i}^{N}\left(V^{\sigma}\right) t+e_{i}^{G}\left(U_{\sigma}^{N}\right)\right) .
$$

Proof. We compute:

$$
\begin{aligned}
& \left.|G| \prod_{i=1}^{r} \frac{1+x^{e_{i}^{G}\left(U_{\sigma}^{N}\right)} y^{e_{i}^{N}\left(V^{\sigma}\right)} u}{1-x^{d_{i}^{G}}}\right|_{\substack{y=x^{t} \\
u=q t(1-x)-1 \\
x \rightarrow 1}} \\
& =|G| \lim _{x \rightarrow 1} \prod_{i=1}^{r}\left(\frac{x^{e_{i}^{G}\left(U_{\sigma}^{N}\right)+t e_{i}^{N}\left(V^{\sigma}\right)} q t(1-x)}{1-x^{d_{i}^{G}}}+\frac{1-x^{e_{i}^{G}\left(U_{\sigma}^{N}\right)+t e_{i}^{N}\left(V^{\sigma}\right)}}{1-x^{d_{i}^{G}}}\right) \\
& =\prod_{i=1}^{r}\left(q t+e_{i}^{N}\left(V^{\sigma}\right) t+e_{i}^{G}\left(U_{\sigma}^{N}\right)\right)
\end{aligned}
$$

Our argument for the sum side of Theorem 1.4 is more delicate. The reason for this is that for $g \in G$ the fixed space of $g$ acting on $U_{\sigma}^{N}$ often has larger dimension than the fixed space of $g$ acting on $V^{\sigma}$, which causes many terms in the term-by-term limit to be zero.

It turns out, as we will now show, that the contributions are correct when taken coset by coset. For this, let us define for each coset $N g \in H=G / N$ the twisted Poincaré series

$$
\mathcal{P}_{\sigma}^{N g}(x, y, u):=\frac{1}{|N|} \sum_{\substack{n \in N \\ \ell, e, p \geq 0}} \operatorname{tr}\left(n g \mid\left(S\left(V^{*}\right)_{\ell} \otimes\left(\bigwedge^{p}\left(U_{\sigma}^{N}\right)^{*}\right)_{e}\right)\right) x^{\ell} y^{e} u^{p},
$$

so that $\mathcal{P}_{\sigma}^{G}(x, y, u)=\frac{1}{|H|} \sum_{N g \in H} \mathcal{P}_{\sigma}^{N g}(x, y, u)$. The following result is proved along the same lines as Proposition 3.16 and serves as an equivalent definition of $\mathcal{P}_{\sigma}^{N g}(x, y, u)$.

Lemma 4.6. $\mathcal{P}_{\sigma}^{N g}(x, y, u)=\frac{1}{|N|} \sum_{n \in N} \frac{\prod_{e \in \mathcal{E}_{\sigma}^{N}} \operatorname{det}\left(1+\left.u y^{e}(n g)\right|_{\left(U_{\sigma}^{N}\right)_{e}^{*}}\right)}{\operatorname{det}\left(1-\left.x(n g)\right|_{V^{*}}\right)}$. 
Proof. For each $n \in N$,

$$
\begin{aligned}
\sum_{\ell, e, p \geq 0} \operatorname{tr}\left(n g \mid\left(S\left(V^{*}\right)_{\ell} \otimes\left(\bigwedge^{p}\left(U_{\sigma}^{N}\right)^{*}\right)_{e}\right)\right) x^{\ell} y^{e} u^{p} \\
=\left(\sum_{\ell \geq 0} \operatorname{tr}\left(n g \mid S\left(V^{*}\right)_{\ell}\right) x^{\ell}\right) \cdot \prod_{e \in \mathcal{E}_{\sigma}^{N}}\left(\sum_{p \geq 0} \operatorname{tr}\left(n g \mid \bigwedge^{p}\left(U_{\sigma}^{N}\right)_{e}^{*}\right) y^{e p} u^{p}\right),
\end{aligned}
$$

and our result follows after taking the average over $n \in N$.

Definition 4.7. We will adopt the following notation for the rest of this section. Let $g \in G$. We will denote by $\bar{\lambda}_{1}(g), \ldots, \bar{\lambda}_{r}(g)$ the set of eigenvalues of $g$ on $V^{*}$. We choose once and for all: a $g$-eigenbasis of fundamental $N$-invariants $N_{i} \in E^{*}$ such that $\operatorname{deg}_{\mathbf{x}}\left(N_{i}\right)=d_{i}^{N}$ and $g N_{i}=\epsilon_{i}^{g}(E) N_{i}$ and a $g$-eigenbasis for the Orlik-Solomon space (see Definition 2.9) $u_{i}^{N} \in\left(U_{\sigma}^{N}\right)^{*}$ such that $\operatorname{deg}_{\mathbf{x}}\left(u_{i}^{N}\right)=e_{i}^{N}\left(V^{\sigma}\right)$ and $g u_{i}^{N}=\epsilon_{i}^{g}\left(U_{\sigma}^{N}\right) u_{i}^{N}$. We observe as in [7] that the multisets of pairs

$$
\left\{\left(\epsilon_{i}^{g}(E), d_{i}^{N}\right) \mid i=1, \ldots, r\right\} \quad \text { and } \quad\left\{\left(\epsilon_{i}^{g}\left(U_{\sigma}^{N}\right), e_{i}^{N}\left(V^{\sigma}\right)\right) \mid i=1, \ldots, r\right\}
$$

depend only on $\sigma$ and the coset $N g \in H$ and not on the choice of coset representative $g \in N g$.

Proposition 4.8. $\mathcal{P}_{\sigma}^{N g}(x, y, u)=\prod_{i=1}^{r} \frac{1+\epsilon_{i}^{g}\left(U_{\sigma}^{N}\right) u y^{e_{i}^{N}\left(V^{\sigma}\right)}}{1-\epsilon_{i}^{g}(E) x^{d_{i}^{N}}}$.

Proof. We write $\mathcal{P}_{\sigma}^{N g}(x, y, u)$ as in Lemma 4.6. First observe that, because $U_{\sigma}^{N}$ is $N$ invariant, for any $n \in N$ we have that

$$
\prod_{e \in \mathcal{E}_{\sigma}^{N}} \operatorname{det}\left(1+u y^{e}\left(\left.n g\right|_{\left(U_{\sigma}^{N}\right)_{e}^{*}}\right)\right)=\prod_{i=1}^{r}\left(1+\epsilon_{i}^{g}\left(U_{\sigma}^{N}\right) u y_{i}^{e_{i}^{N}\left(V^{\sigma}\right)}\right),
$$

independently of $n \in N$.

Let $\mathcal{D}_{N}=\left\{d_{1}^{N}, \ldots, d_{r}^{N}\right\}$ denote the set of degrees for $N$, and let $E_{d}^{*}=\operatorname{span}_{\mathbb{C}} \mathbf{N}_{d}$ for $d \in \mathcal{D}_{N}$, where as before $\mathbf{N}_{d}$ denotes the set of fundamental $N$-invariants having degree d. Because $S\left(E^{*}\right) \simeq \bigotimes_{d \in \mathcal{D}_{N}} S\left(E_{d}^{*}\right)$, we have that

$$
\sum_{\ell \geq 0} \operatorname{tr}\left(g \mid S\left(E^{*}\right)_{\ell}\right) x^{\ell}=\prod_{d \in \mathcal{D}_{N}}\left(\sum_{\ell \geq 0}\left(\operatorname{tr}\left(g \mid \operatorname{Sym}^{\ell}\left(E_{d}^{*}\right)\right)\left(x^{d}\right)^{\ell}\right)\right)
$$

where $S\left(E^{*}\right)_{\ell}:=S\left(E^{*}\right) \cap \operatorname{Sym}^{\ell}\left(V^{*}\right)=\operatorname{Sym}^{\ell}\left(V^{*}\right)^{N}$, and $\operatorname{Sym}^{\ell}\left(V^{*}\right)$ denotes the $\ell$ th symmetric power of $V^{*}$. On the other hand,

$$
\prod_{d \in \mathcal{D}_{N}}\left(\sum_{\ell \geq 0} \operatorname{tr}\left(g \mid \operatorname{Sym}^{\ell}\left(E_{d}^{*}\right)\right)\left(x^{d}\right)^{\ell}\right)=\prod_{d \in \mathcal{D}_{N}} \frac{1}{\operatorname{det}\left(1-x^{d}\left(\left.g\right|_{E_{d}^{*}}\right)\right)}=\prod_{i=1}^{r} \frac{1}{1-\epsilon_{i}^{g}(E) x_{i}^{d_{i}^{N}}} .
$$

Therefore,

$$
\sum_{\ell \geq 0} \operatorname{tr}\left(g \mid S\left(E^{*}\right) \ell\right) x^{\ell}=\prod_{i=1}^{r} \frac{1}{1-\epsilon_{i}^{g}(E) x_{i}^{d_{i}^{N}}}
$$


Because, for $n \in N$,

$$
\sum_{\ell \geq 0} \operatorname{tr}\left(n g \mid \operatorname{Sym}^{\ell}\left(V^{*}\right)\right) x^{\ell}=\frac{1}{\operatorname{det}\left(1-x\left(\left.n g\right|_{V^{*}}\right)\right)},
$$

it remains to show that

$$
\frac{1}{|N|} \sum_{n \in N}\left(\sum_{\ell \geq 0} \operatorname{tr}\left(n g \mid \operatorname{Sym}^{\ell}\left(V^{*}\right)\right) x^{\ell}\right)=\sum_{\ell \geq 0} \operatorname{tr}\left(g \mid S\left(E^{*}\right)_{\ell}\right) x^{\ell}
$$

or, equivalently, that for each $\ell \geq 0$ we have that

$$
\frac{1}{|N|} \sum_{n \in N}\left(\operatorname{tr}\left(n g \mid \operatorname{Sym}^{\ell}\left(V^{*}\right)\right)\right)=\operatorname{tr}\left(g \mid S\left(E^{*}\right)_{\ell}\right) .
$$

To see this, note that the operator on $\operatorname{Sym}^{\ell}\left(V^{*}\right)$ given by

$$
\frac{1}{|N|} \sum_{n \in N} n g=g \cdot\left(\frac{1}{|N|} \sum_{n \in N} n\right)=g \circ \operatorname{pr}_{\ell}^{N}
$$

where $\operatorname{pr}_{\ell}^{N}=\frac{1}{|N|} \sum_{n \in N} n$ is the projection from $\operatorname{Sym}^{\ell}\left(V^{*}\right)$ onto its $g$-stable subspace $\operatorname{Sym}^{\ell}\left(V^{*}\right)^{N}=S\left(E^{*}\right)_{\ell}$, whence $\operatorname{tr}\left(\left(g \circ \operatorname{pr}_{\ell}^{N}\right) \mid \operatorname{Sym}^{\ell}\left(V^{*}\right)\right)=\operatorname{tr}\left(g \mid S\left(E^{*}\right)_{\ell}\right)$.

Proposition 4.9 ([7, Theorem 3.1]).

$$
\frac{1}{|N|} \sum_{n \in N} \frac{\operatorname{det}\left(1+\left.u(n g)\right|_{\left(V^{\sigma}\right)^{*}}\right)}{\operatorname{det}\left(1-\left.x(n g)\right|_{V^{*}}\right)}=\prod_{i=1}^{r} \frac{1+\epsilon_{i}^{g}\left(U_{\sigma}^{N}\right) u x^{e_{i}^{N}\left(V^{\sigma}\right)}}{1-\epsilon_{i}^{g}(E) x^{d_{i}^{N}}}=\mathcal{P}_{\sigma}^{N g}(x, x, u),
$$

Proof. The first equality is a special case of [7, Theorem 3.1] (where we note that the change in sign from the $-u$ in their notation to $+u$ in our notation is harmless), and the second equality follows directly from Proposition 4.8 .

We obtain the following crucial specialisation of Proposition 4.8, which exploits the similarity between Proposition 4.9 and Proposition 4.8 and is inspired by [7, Theorem 3.3]

Proposition 4.10. For $g \in G$, with notation as in Definition 4.7,

$$
\lim _{x \rightarrow 1}|N| \cdot \mathcal{P}_{\sigma}^{N g}\left(x, x^{t}, q t(1-x)-1\right)=t^{\mathrm{fix} x_{E}(g)} \sum_{n \in N}\left(\prod_{\bar{\lambda}_{i}(n g) \neq 1} \frac{1-\bar{\lambda}_{i}(n g)^{\sigma}}{1-\bar{\lambda}_{i}(n g)}\right) q^{\mathrm{fix}_{V}(n g)} .
$$

Proof. Let us agree to index the pairs $\left(\epsilon_{i}^{g}(E), d_{i}^{N}\right)$ and $\left(\epsilon_{i}^{g}\left(U_{\sigma}^{N}\right), e_{i}^{N}\left(V^{\sigma}\right)\right)$ in the multisets from Definition 4.7 such that $\epsilon_{i}^{g}(E)=1$ for $1 \leq i \leq \operatorname{fix}_{E}(g)($ if fix $E(g) \neq 0)$ and $\epsilon_{i}^{g}\left(U_{\sigma}^{N}\right)=1$ for $1 \leq i \leq \operatorname{fix}_{U_{\sigma}^{N}}(g)$ (if $\operatorname{fix}_{U_{\sigma}^{N}}(g) \neq 0$ ). By Proposition 4.8, the left-hand side of Equation (11) is

$$
|N| \cdot \lim _{x \rightarrow 1} \prod_{i=1}^{r}\left(\frac{\epsilon_{i}^{g}\left(U_{\sigma}^{N}\right) q t(1-x) x^{t e_{i}^{N}\left(V^{\sigma}\right)}}{1-\epsilon_{i}^{g}(E) x^{d_{i}^{N}}}+\frac{1-\epsilon_{i}^{g}\left(U_{\sigma}^{N}\right) x^{t e_{i}^{N}\left(V^{\sigma}\right)}}{1-\epsilon_{i}^{g}(E) x^{d_{i}^{N}}}\right)
$$


By [7, Proposition 3.2], fix $\operatorname{U}_{\sigma}^{N}(g) \geq$ fix $_{E}(g)$. We will compute the above limit for the partial products ranging over $1 \leq i \leq \operatorname{fix}_{E}(g)$, fix $x_{E}(g)+1 \leq i \leq \operatorname{fix}_{U_{\sigma}^{N}}(g)$ and $\operatorname{fix}_{U_{\sigma}^{N}}(g)+1 \leq i \leq r$ separately.

Because $\epsilon_{i}^{g}(E)=1=\epsilon_{i}^{g}\left(U_{\sigma}^{N}\right)$ for $1 \leq i \leq \operatorname{fix}_{E}(g)$, we have that

$$
\lim _{x \rightarrow 1} \prod_{i=1}^{\mathrm{fix}_{E}(g)} \frac{q t(1-x) x^{t e_{i}^{N}\left(V^{\sigma}\right)}}{1-x^{d_{i}^{N}}}+\frac{1-x^{t e_{i}^{N}\left(V^{\sigma}\right)}}{1-x^{d_{i}^{N}}}=\prod_{i=1}^{\mathrm{fix}_{E}(g)} \frac{q t+t e_{i}^{N}\left(V^{\sigma}\right)}{d_{i}^{N}} .
$$

Because $\epsilon_{i}^{g}(E) \neq 1 \neq \epsilon_{i}^{g}\left(U_{\sigma}^{N}\right)$ for $\operatorname{fix}_{U_{\sigma}^{N}}(g)+1 \leq i \leq r$, we have that

$$
\lim _{x \rightarrow 1} \prod_{i=\operatorname{fix}_{U_{\sigma}^{N}}^{r}(g)+1}^{r}\left(\frac{1+\epsilon_{i}^{g}\left(U_{\sigma}^{N}\right)(q t(1-x)-1) x^{t e_{i}^{N}\left(V^{\sigma}\right)}}{1-\epsilon_{i}^{g}(E) x^{d_{i}^{N}}}\right)=\prod_{i=\operatorname{fix}_{U_{\sigma}^{N}}(g)+1}^{r} \frac{1-\epsilon_{i}^{g}\left(U_{\sigma}^{N}\right)}{1-\epsilon_{i}^{g}(E)} .
$$

If the inequality $\operatorname{fix}_{U_{\sigma}^{N}}(g)>\operatorname{fix}_{E}(g)$ is strict, so that $\epsilon_{i}^{g}(E) \neq 1=\epsilon_{i}^{g}\left(U_{\sigma}^{N}\right)$ for fix $\operatorname{fin}_{E}(g)+1 \leq$ $i \leq \operatorname{fix}_{U_{\sigma}^{N}}(g)$, then we see that for each such $i$ the limit of the corresponding factor is

$$
\lim _{x \rightarrow 1} \frac{1+(q t(1-x)-1) x^{t e_{i}^{N}\left(V^{\sigma}\right)}}{1-\epsilon_{i}^{g}(E) x^{d_{i}^{N}}}=0 .
$$

Therefore, if $\operatorname{fix}_{U_{\sigma}^{N}}(g)>\operatorname{fix}_{E}(g)$, then (cf. [7, Theorem 3.3])

$$
\lim _{x \rightarrow 1}|N| \cdot \mathcal{P}_{\sigma}^{N g}\left(x, x^{t}, q t(1-x)-1\right)=0 .
$$

On the other hand, if $\operatorname{fix}_{U_{\sigma}^{N}}(g)=\operatorname{fix}_{E}(g)$, then (cf. [7, Theorem 3.3])

$$
\lim _{x \rightarrow 1}|N| \cdot \mathcal{P}_{\sigma}^{N g}\left(x, x^{t}, q t(1-x)-1\right)=t^{\mathrm{fix} E}(g) \prod_{i=1}^{\mathrm{fix}_{E}(g)}\left(q+e_{i}^{N}\left(V^{\sigma}\right)\right) \prod_{i=\operatorname{fix}_{E}(g)+1}^{r} \frac{1-\epsilon_{i}^{g}\left(U_{\sigma}^{N}\right)}{1-\epsilon_{i}^{g}(E)} d_{i}^{N} .
$$

In any case, we have shown that the left-hand side of Equation $(11)$ is $t^{\mathrm{fix}}(g) \cdot P(q)$ for some $P(q) \in \mathbb{C}[q]$. To conclude the proof, it suffices to compare the left- and right-hand sides of Equation (11) at $t=1$. For this, we observe as in [7, Theorem 3.3] that, as a consequence of Proposition 4.9 and the arguments of [16, Theorem 3.3] that are now standard,

$$
\begin{aligned}
\lim _{x \rightarrow 1}|N| \cdot \mathcal{P}_{\sigma}^{N g}(x, x, q(1-x)-1) & =\left.\sum_{n \in N}\left(\prod_{i=1}^{r} \frac{1+\bar{\lambda}_{i}(n g)^{\sigma} u}{1-\bar{\lambda}_{i}(n g) x}\right)\right|_{\substack{u=q(1-x)-1 \\
x \rightarrow 1}} \\
& =\sum_{n \in N}\left(\prod_{\bar{\lambda}_{i}(n g) \neq 1} \frac{1-\bar{\lambda}_{i}(n g)^{\sigma}}{1-\bar{\lambda}_{i}(n g)}\right) q^{\mathrm{fix}_{V}(n g)} .
\end{aligned}
$$

Corollary 4.11 (Sum side specialisation).

$$
\lim _{x \rightarrow 1}|G| \cdot \mathcal{P}_{\sigma}^{G}\left(x, x^{t}, q t(1-x)-1\right)=\sum_{g \in G}\left(\prod_{\lambda_{i}(g) \neq 1} \frac{1-\lambda_{i}(g)^{\sigma}}{1-\lambda_{i}(g)}\right) q^{\mathrm{fix}_{V}(g)} t^{\mathrm{fix}_{E}(g)} .
$$


Proof. Let $g_{1}, \ldots, g_{|H|} \in G$ be a full set of coset representatives for $G / N=H$. Because $\mathcal{P}_{\sigma}^{G}(x, y, u)=\frac{1}{|H|} \sum_{j=1}^{|H|} \mathcal{P}_{\sigma}^{N g_{j}}(x, y, u)$ and $\operatorname{fix}_{E}\left(g_{j}\right)=$ fix $_{E}\left(n g_{j}\right)$ for any $n \in N$, it follows from Proposition 4.10 that

$$
\begin{aligned}
\lim _{x \rightarrow 1}|G| \cdot \mathcal{P}_{\sigma}^{G} & \left(x, x^{t}, q t(1-x)-1\right)=\sum_{j=1}^{|H|} \lim _{x \rightarrow 1}|N| \cdot \mathcal{P}_{\sigma}^{N g_{j}}\left(x, x^{t}, q t(1-x)-1\right)= \\
& =\sum_{j=1}^{|H|} t^{\mathrm{fix}_{E}\left(g_{j}\right)} \sum_{n \in N}\left(\prod_{\bar{\lambda}_{i}\left(n g_{j}\right) \neq 1} \frac{1-\bar{\lambda}_{i}\left(n g_{j}\right)^{\sigma}}{1-\bar{\lambda}_{i}\left(n g_{j}\right)}\right) q^{\mathrm{fix}_{V}\left(n g_{j}\right)} \\
& =\sum_{g \in G}\left(\prod_{\bar{\lambda}_{i}(g) \neq 1} \frac{1-\bar{\lambda}_{i}(g)^{\sigma}}{1-\bar{\lambda}_{i}(g)}\right) q^{\mathrm{fix} V(g)} t^{\mathrm{fix}_{E}(g)},
\end{aligned}
$$

and our result follows after replacing $g$ with $g^{-1}$.

Remark 4.12. As mentioned in the Introduction, the formula of Theorem 1.4 corresponding to the special case $\sigma=1$ becomes [1, Theorem 1.5]

$$
\sum_{g \in G} q^{\mathrm{fix}_{V}(g)} t^{\mathrm{fix}_{E}(g)}=\prod_{i=1}^{r}\left(q t+e_{i}^{N}(V) t+e_{i}^{G}(E)\right),
$$

which recovers Equation (2) for the reflection group $G$ by evaluating at $t=1$, because $E \simeq$ $U^{N}$ as $G$-modules in this case by Lemma 4.1 and $e_{i}^{N}(V)+e_{i}^{G}(E)=e_{i}^{G}(V)$ by Theorem 1.3, as discussed in Remark 4.2.

On the other hand, specialising Equation (12) at $q=1$ and dividing by $|N|$ on both sides again recovers Equation (2) but this time for the reflection group $H$ : the sum side follows from observing that $\operatorname{fix}_{E}(N g)=\operatorname{fix}_{E}(g)$ for every $N g \in H$. The product side follows from the equality $d_{i}^{N} \cdot e_{i}^{H}(E)=e_{i}^{G}(E)$ proved in Theorem 1.3, which is compatible with the classical identities $1+e_{i}^{N}(V)=d_{i}^{N}$ and $\prod_{i=1}^{r} d_{i}^{N}=|N|$ by Remark 4.2.

In fact, it is also possible to recover Equation (2) for the reflection group $N$ from Equation (12). Because $H$ acts faithfully on $E$, we have $N=\left\{g \in G \mid \operatorname{fix}_{E}(g)=r\right\}$, and therefore applying $\frac{1}{r !} \frac{\partial^{r}}{\partial t^{r}}$ to the sum-side of Equation (12) recovers the sum side of Equation (2) for $N$. That the analogous result obtains for the product side follows from the well-known higher Leibniz rule for the Hasse-Schmidt derivations $\delta^{(i)}:=\frac{1}{i !} \frac{\partial^{i}}{\partial t^{i}}$, which yield

$$
\delta^{(r)}\left(\prod_{i=1}^{r}\left(q t+e_{i}^{N}(V) t+e_{i}^{G}(E)\right)\right)=\prod_{i=1}^{r} \delta^{(1)}\left(q t+e_{i}^{N}(V) t+e_{i}^{G}(E)\right)=\prod_{i=1}^{r}\left(q+e_{i}^{N}(V)\right) .
$$

Similarly, as we mentioned in the Introduction, for arbitrary $\sigma \in \operatorname{Gal}\left(\mathbb{Q}\left(\zeta_{G}\right) / \mathbb{Q}\right)$ the formula of Theorem 1.4

$$
\sum_{g \in G}\left(\prod_{\lambda_{i}(g) \neq 1} \frac{1-\lambda_{i}(g)^{\sigma}}{1-\lambda_{i}(g)}\right) q^{\mathrm{fix}_{V}(g)} t^{\mathrm{fix}_{E}(g)}=\prod_{i=1}^{r}\left(q t+e_{i}^{N}\left(V^{\sigma}\right) t+e_{i}^{G}\left(U_{\sigma}^{N}\right)\right)
$$


recovers Theorem 1.1 by evaluating at $t=1$, because $e_{i}^{N}\left(V^{\sigma}\right)+e_{i}^{G}\left(U_{\sigma}^{N}\right)=e_{i}^{G}\left(V^{\sigma}\right)$ by Theorem 1.3. The above arguments also show that we recover Theorem 1.1 for the reflection group $N$ again by applying $\frac{1}{r !} \frac{\partial^{r}}{\partial t^{r}}$ to both sides of Equation (13).

It would be desirable to recover Theorem 1.1 for the reflection group $H$ from Theorem 1.4 in analogy with the case $\sigma=1$, by evaluating Equation (13) at $q=1$ and dividing by $|N|$ on both sides. But in this case we obtain something else: letting $g_{1}, \ldots, g_{|H|} \in G$ be a full set of coset representatives for $H=G / N$, evaluating Equation (13) at $q=1$ yields

$$
\sum_{j=1}^{|H|}\left(\sum_{n \in N}\left(\prod_{\lambda_{i}\left(n g_{j}\right) \neq 1} \frac{1-\lambda_{i}\left(n g_{j}\right)^{\sigma}}{1-\lambda_{i}\left(n g_{j}\right)}\right)\right) t^{\mathrm{fix}_{E}\left(g_{j}\right)}=\prod_{i=1}^{r}\left(\left(e_{i}^{N}\left(V^{\sigma}\right)+1\right) t+e_{i}^{G}\left(U_{\sigma}^{N}\right)\right),
$$

which does not immediately compare to the statement of Theorem 1.1 for the reflection group $H$ :

$$
\sum_{j=1}^{|H|}\left(\prod_{\bar{\epsilon}_{i}^{g_{j}}(E) \neq 1} \frac{1-\bar{\epsilon}_{i}^{g_{j}}(E)^{\sigma}}{1-\bar{\epsilon}_{i}^{g_{j}}(E)}\right) t^{\mathrm{fix}_{E}\left(g_{j}\right)}=\prod_{i=1}^{r}\left(t+e_{i}^{H}\left(E^{\sigma}\right)\right),
$$

where compatibly with Definition 4.7 the $\bar{\epsilon}_{i}^{g_{j}}(E)$ denote the eigenvalues of $g_{j} \in G$ acting on $E$.

\section{Reflexponents revisited}

Fix $G$ a complex reflection group of rank $r$ with reflection representation $V$. Call an $r$-dimensional representation $M$ of $G$ factorising if $M$ has dimension $r$ and

$$
\sum_{g \in G} q^{\mathrm{fix}_{V}(g)} t^{\mathrm{fix}_{M}(g)}=\prod_{i=1}^{r}\left(q t+\left(e_{i}^{G}(V)-m_{i}\right) t+m_{i}\right)
$$

for some nonnegative integers $m_{1}, \ldots, m_{r}$. More generally, call a representation $M$ of $G$ of dimension $\operatorname{dim} M \leq r$ factorising if it is factorising in the above sense after adding in $r-\operatorname{dim} M$ copies of the trivial representation.

A case-by-case construction of a factorising representation $M_{\mathcal{H}}$ associated to an arbitrary orbit of reflecting hyperplanes $\mathcal{H}$ was presented in [22], with two unexplained exceptions. These factorising representations further restricted to the reflection representation of a parabolic subgroup supported on $\mathcal{H}$. We can now give a uniform explanation for those ad hoc identities, including the two exceptions left unexplained in [22, Section 5.1].

Let $\mathcal{H}$ be an orbit of hyperplanes, write $\mathcal{R}_{\mathcal{H}}$ for the set of reflections fixing some $H \in \mathcal{H}$ and let $N_{\mathcal{H}}=\left\langle\mathcal{R}_{\mathcal{H}}\right\rangle$ be the subgroup generated by reflections around hyperplanes in $\mathcal{H}$. Because these reflections form a conjugacy class in $G, N_{\mathcal{H}}$ is a normal reflection subgroup of $G$. Furthermore,

(i) the quotient $G / N_{\mathcal{H}}$ acts by reflections on the vector space of $N_{\mathcal{H}}$-orbits $M_{\mathcal{H}}$,

(ii) this $G$-representation $M_{\mathcal{H}}$ is factorising by Theorem 1.4 and 
(iii) the mysterious indexing of the reflexponents (i.e., the $\left.e_{i}^{G}\left(M_{\mathcal{H}}\right)\right)$ left unexplained in [22] is now explained in Remark 3.1.

Corollary 5.1. For $\mathcal{H}$ an orbit of hyperplanes and $N_{\mathcal{H}}=\left\langle\mathcal{R}_{\mathcal{H}}\right\rangle$, the representation $M_{\mathcal{H}}$ is factorising.

We can also explain the two exceptional factorising representations from [22]:

- Following the conventions of [15], $G=G_{13}=\langle s, t, u\rangle$ was observed in [22, Section 5.1] to have a 2-dimensional representation with the factorising property. The group $N=\left\langle g s g^{-1}: g \in G\right\rangle$ (fixing the conjugacy class $\mathcal{H}_{s}$ ) is a normal subgroup isomorphic to $G(4,2,2)$ and the quotient $G / N \simeq W\left(A_{2}\right) \simeq \mathfrak{S}_{3}$ gives the unexplained 2-dimensional factorising representation in this case.

- For $G=G(a b, b, r)=\left\langle s, t_{2}, t_{2}^{\prime}, t_{3}, \ldots, t_{r}\right\rangle$ with $a, b>1$ and $r>2$, we can take $N=$ $\left\langle g s g^{-1}: g \in G\right\rangle . N$ is a normal subgroup of $G$ isomorphic to $\left(C_{a}\right)^{r}$ (it consists of diagonal matrices whose diagonal entries are ath roots of unity). The quotient $G / N \simeq G(b, b, r)$ gives the unexplained $r$-dimensional factorising representation in $[22$, Section 5.2].

\section{Classification of Normal Reflection Subgroups}

In this section, we state the classification of normal reflection subgroups of irreducible complex reflection groups.

Recall that $G(a b, b, r)$ is given in its standard reflection representation as the set of $r \times r$ monomial matrices whose every nonzero entry is an $(a b)$ th root of unity and in which the product of the nonzero entries is an ath root of unity. The following theorem identifies the normal reflection subgroups of the infinite family $G(a b, b, r)$.

Theorem 6.1 ([14, Chapter 2]). Fix positive integers $a$ and $b$, and let $a=$ de.

For rank $r=1, G(a b, b, 1)=C_{a}$ has normal subgroups and quotients

(1.a) $C_{a} / C_{d} \simeq C_{e}$.

For rank $r=2$, the normal subgroups and quotients are

(2.a) $G(a b, b, 2) /\left(C_{d}\right)^{2} \simeq G(e b, b, 2)$,

(2.b) $G(a b, b, 2) / G(a b, d b, 2) \simeq C_{d}$,

(2.c) $G(2 a b, 2 d, 2) / G(a b, a, 2) \simeq C_{2} \times C_{e}$.

For rank $r \geq 3$, the normal subgroups and quotients are

(r.a) $G(a b, b, r) /\left(C_{d}\right)^{r} \simeq G(e b, b, r)$ and

(r.b) $G(a b, b, r) / G(a b, d b, r) \simeq C_{d}$.

In cases (r.a) for $r \geq 1$, the polycyclic group $N=\left(C_{d}\right)^{r}$ is included in $G$ as diagonal matrices with each nonzero entry a $d$ th root of unity. In cases $(r . b)$ for $r \geq 2$, the normal reflection subgroup $N=G(a b, d b, r)$ is included in $G$ via its standard reflection representation on $\mathbb{C}^{r}$. In case (2.c), the normal reflection subgroup $N=G(a b, a, 2)$ occurs twice in $G=G(2 a b, 2 d, 2)$ : once via its standard reflection representation, and once as the 
group generated by the reflections $\left\{\operatorname{diag}\left(\zeta_{b}^{k}, 1\right), \operatorname{diag}\left(1, \zeta_{b}^{k}\right) \mid k=1, \ldots, b-1\right\}($ when $b \neq 1)$ along with the reflections

$$
\left\{\left(\begin{array}{cc}
0 & \zeta_{2 a b}^{2 k-1} \\
\zeta_{2 a b}^{-2 k+1} & 0
\end{array}\right) \mid k=0, \ldots, a b-1\right\} .
$$

Independent of the factorization $a=d e$, these two copies of $N$ are conjugate in $\mathrm{GL}_{2}(\mathbb{C})$ by the matrix $\operatorname{diag}\left(1, \zeta_{2 a b}\right)$, which normalizes $G$. There are other coincidences in rank $r=2$ where $G$ contains several copies of 'the same' normal reflection subgroup $N$, related to the well-known isomorphisms $G(4,4,2) \simeq G(2,1,2)$ and $G(2,2,2) \simeq C_{2} \times C_{2}$ (see [14, Example $2.11])$. For example, $G(4,2,2)$ contains the dihedral group of order 8 as a normal reflection subgroup in three different ways: $G(4,4,2)$ in one way as case $(2$.b) and $G(2,1,2)$ in two ways as case (2.c). Similarly, $G(4,2,2)$ contains $C_{2} \times C_{2}$ in three ways: $\left(C_{2}\right)^{2}$ in one way as case (2.a) and $G(2,2,2)$ in two ways as case (2.c).

The exceptional (that is, primitive) irreducible complex reflection groups $G$ and their normal reflection subgroups $N$ are listed in Table 1 . This classification was computed with Sage [21] using the code available at [2]. Most examples occur in rank $r=2$. In rank $r \geq 3$, every reflection has order 2 or 3 [14, Theorem 8.4] and the only exceptional groups with more than a single orbit of reflections are $G_{26}$ and $G_{28}$ [14, Table D.2], which leads to the four nontrivial exceptional examples listed in Table 1 in rank $r \geq 3$.

An isomorphism type of $N$ is unique up to conjugation in $\mathrm{GL}_{r}(\mathbb{C})$ sending isomorphic normal reflection subgroups to each other while stabilising the reflection representation of $G$. In fact, there are only two instances in the exceptional groups $\left(G_{5} \triangleright G_{4}\right.$ and $G_{28} \triangleright$ $G(2,2,4))$ where an isomorphism type appears as a normal subgroup more than once. We gather the situations where the same isomorphism type of $N$ occurs more than once as a normal reflection subgroup of $G$ in the following result, where $N_{(i)}$ denote the different isomorphic copies of the same normal reflection subgroup $N \triangleleft G$.

Theorem 6.2. Suppose that $G$ is an irreducible complex reflection group admitting normal reflection subgroups $N_{(1)}, \ldots, N_{(k)}$ for $k \geq 2$ that are pairwise isomorphic (as abstract groups) but not equal in $G$. Then $k \in\{2,3\}$ and the normaliser of $G$ in $\mathrm{GL}(V)$ permutes $\left\{N_{(1)}, \ldots, N_{(k)}\right\}$ transitively under conjugation. Moreover, precisely one of the following possibilities occurs:

(1) $G=G(4,2,2)$ and there are $k=3$ different normal reflection subgroups isomorphic to the dihedral group of order 8 :

(a) $N_{(1)}=G(2,1,2)$ as in Theorem 6.1(2.c);

(b) $N_{(2)}=\operatorname{diag}(1,-i) \cdot N_{(1)} \cdot \operatorname{diag}(1, i)$;

(c) $N_{(3)}=G(4,4,2)$ as in Theorem 6.1(2.b).

(2) $G=G(4,2,2)$ and there are $k=3$ different normal reflection subgroups isomorphic to the Klein 4-group:

(a) $N_{(1)}=G(2,2,2)$ as in Theorem 6.1(2.c);

(b) $N_{(2)}=\operatorname{diag}(1,-i) \cdot N_{(1)} \cdot \operatorname{diag}(1, i)$;

(c) $N_{(3)}=\left(C_{2}\right)^{2}$ as in Theorem 6.1(2.a). 
TABLE 1. The exceptional groups, their nontrivial normal reflection subgroups and the corresponding quotient reflection groups.

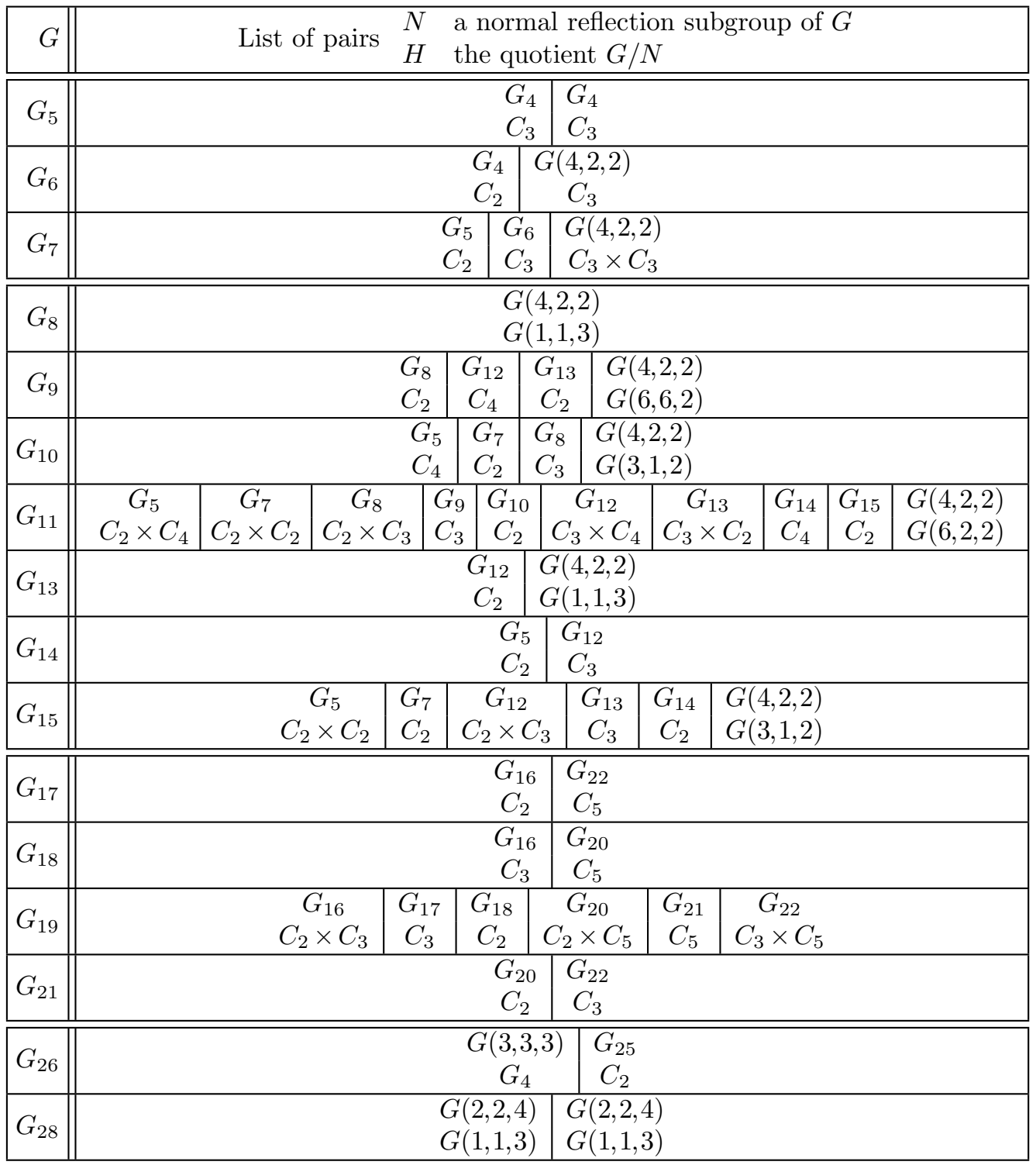

(3) $G=G(2 a b, 2 d, 2) \neq G(4,2,2)$ with $d$ a factor of a and there are $k=2$ different normal reflection subgroups isomorphic to $G(a b, a, 2)$ :

(a) $N_{(1)}=G(a b, a, 2)$ as in Theorem 6.1(2.c);

(b) $N_{(2)}=\operatorname{diag}\left(1, \zeta_{2 a b}^{-1}\right) \cdot N_{(1)} \cdot \operatorname{diag}\left(1, \zeta_{2 a b}\right)$. 
(4) $G=G_{5}$ and there are $k=2$ different normal reflection subgroups isomorphic to $G_{4}$.

(5) $G=G_{28}$ and there are $k=2$ different normal reflection subgroups isomorphic to $G(2,2,4)$.

Proof. By Theorem 6.1 and Table 1, the possibilities above are exhaustive, and it is clear that they are mutually exclusive.

It remains to show that any two isomorphic normal reflection subgroups of $G$ are conjugate under an element of the normaliser of $G$. In every situation listed above, except for $G=G_{28}$, we can find a complex reflection group $W$ that contains $G$ as a normal reflection subgroup (in its standard reflection representation) but that does not contain (the isomorphism type of) $N$ as a normal reflection subgroup. Because there are at most three isomorphic copies of $N$ in each case, they must then form a single conjugacy class in $W$.

In cases (1) and (2), we can take $W$ to be any of $G_{6}, G_{7}, G_{8}, G_{9}, G_{10}, G_{11}, G_{13}$ or $G_{15}$ because all of these contain $G=G(4,2,2)$ as their only imprimitive normal reflection subgroup according to Table 1.

In case (3), we can take $W=G(2 a b, 1,2)$, which contains $G=G(2 a b, 2 d, 2)$ as a normal reflection subgroup as in Theorem 6.1(2.b) for each factor $d$ of $a$, but does not normalise $N=G(a b, a, 2)$.

In case (4), we can take $W$ to be any of $G_{7}, G_{10}, G_{11}, G_{14}$ or $G_{15}$ because all of these contain $G_{5}$ as a normal reflection subgroup but do not normalise $G_{4}$.

Finally, in case (5) it is not possible to find a complex reflection group $W$ containing $G=G_{28}$ as a normal reflection subgroup because $G_{28}$ is the only irreducible complex reflection group admitting a nontrivial normal reflection subgroup in rank $r \geq 4$. To see that the two isomorphic copies of $N \simeq G(2,2,4)$ in $G=G_{28}$ are conjugate under an element of the normaliser of $G$ in $\mathrm{GL}_{4}(\mathbb{C})$, consider the set of reflecting hyperplanes for $G_{28}$, which are the orthogonal complements (with respect to the standard Hermitian inner product in $\mathbb{C}^{4}$ ) of the lines in $\mathcal{L}_{1} \cup \mathcal{L}_{2} \cup \mathcal{L}_{3}$ defined by (cf. [14, Section 7.6.2]):

$$
\begin{gathered}
\mathcal{L}_{1}=\left\{\mathbb{C} \cdot e_{i} \mid 1 \leq i \leq 4\right\} ; \quad \mathcal{L}_{2}=\left\{\mathbb{C} \cdot \frac{1}{2}\left(e_{1} \pm e_{2} \pm e_{3} \pm e_{4}\right)\right\} ; \quad \text { and } \\
\mathcal{L}_{3}=\left\{\mathbb{C} \cdot\left(e_{i} \pm e_{j}\right) \mid 1 \leq i<j \leq 4\right\},
\end{gathered}
$$

where $e_{i}$ denotes the standard basis vector with 1 in the $i$ th entry and 0 elsewhere. There are two orbits of reflecting hyperplanes for $G_{28}=W\left(F_{4}\right)$ (the Weyl group of type $F_{4}$ and a real reflection group), corresponding to $\mathcal{L}_{1} \cup \mathcal{L}_{2}$ (the lines spanned by the short roots) and $\mathcal{L}_{3}$ (the lines spanned by the long roots). The reflections around the 12 hyperplanes corresponding to $\mathcal{L}_{3}$ generate the normal reflection subgroup $N_{(1)}=G(2,2,4)$ acting in its standard reflection representation. The other normal reflection subgroup $N_{(2)}$ is generated by the reflections around the other 12 hyperplanes corresponding to $\mathcal{L}_{1} \cup \mathcal{L}_{2}$. To conclude the proof, note that the real orthogonal matrix

$$
P:=\frac{1}{\sqrt{2}}\left(\begin{array}{cccc}
0 & 1 & 0 & 1 \\
1 & 0 & 1 & 0 \\
0 & 1 & 0 & -1 \\
-1 & 0 & 1 & 0
\end{array}\right)
$$


exchanges the two $G_{28}$-orbits of reflecting hyperplanes $\mathcal{L}_{1} \cup \mathcal{L}_{2} \leftrightarrow \mathcal{L}_{3}$ (identifying $\mathcal{L}_{1}$ with the set of lines $\mathbb{C} \cdot\left(e_{i} \pm e_{j}\right) \in \mathcal{L}_{3}$ such that $\left.j-i=2\right)$. Hence, $P$ normalises $G_{28}$ and exchanges $N_{(1)}$ and $N_{(2)}$ under conjugation.

\section{Examples}

In this section we illustrate our results with examples, beginning with the cyclic groups in Section 7.1, continuing with the infinite family in Section 7.2 and concluding with a non-well-generated exceptional example in Section 7.3.

\subsection{Cyclic Groups}

Consider $G=C_{a}=\langle c\rangle$, the cyclic group of order $a$, acting on $V=\mathbb{C}$ via $c \mapsto \zeta_{a}^{-1}$, where $\zeta_{a}$ is a primitive $a$ th root of unity. Verifying Theorem 1.1 and determining the Orlik-Solomon space $U_{\sigma}^{G}$ from Definition 2.9 for $\sigma \in \operatorname{Gal}\left(\mathbb{Q}\left(\zeta_{a}\right) / \mathbb{Q}\right)$ is already an interesting calculation in this case.

Let $\sigma \in \operatorname{Gal}\left(\mathbb{Q}\left(\zeta_{a}\right) / \mathbb{Q}\right)$ act as $\sigma: \zeta_{a} \mapsto \zeta_{a}^{s}$, where $s \in \mathbb{N}$ is coprime to a. Although it is sufficient to only consider $s \in\{1, \ldots, a-1\}$, it will be essential to allow more general positive exponents $s$ in the description of the action of $\sigma$ on different roots of unity when we begin considering (normal reflection) subgroups of $G$ shortly. We compute the identity of Theorem 1.1 in this example:

$$
\begin{aligned}
\sum_{g \in G}\left(\prod_{\lambda_{1}(g) \neq 1} \frac{1-\lambda_{1}(g)^{\sigma}}{1-\lambda_{1}(g)}\right) q^{\mathrm{fix}_{V}(g)} & =q+\sum_{k=1}^{a-1} \frac{1-\zeta_{a}^{k s}}{1-\zeta_{a}^{k}}=q+\sum_{k=1}^{a-1} \sum_{j=0}^{s-1} \zeta_{a}^{k j} \\
& =q+\left(\sum_{j=0}^{s-1} \sum_{k=0}^{a-1} \zeta_{a}^{k j}\right)-s \\
& =q+\left(\sum_{j=0}^{s-1}\left\{\begin{array}{ll}
a & \text { if } a \mid j \\
0 & \text { otherwise }
\end{array}\right)-s\right. \\
& =q+a\left\lceil\frac{s}{a}\right\rceil-s .
\end{aligned}
$$

On the other hand, we verify that $e_{i}^{G}\left(V^{\sigma}\right)=a\left\lceil\frac{s}{a}\right\rceil-s$ by exhibiting $x^{a\left\lceil\frac{s}{a}\right\rceil-s} \otimes x^{\sigma}$ as a basis vector for the dual $\left(U_{\sigma}^{G}\right)^{*}$ of the Orlik-Solomon space $U_{\sigma}^{G}$, where $x$ and $x^{\sigma}$ denote basis vectors for $V^{*}$ and $\left(V^{\sigma}\right)^{*}$, respectively. More generally, we have the following.

Lemma 7.1. For $G=C_{a}=\langle c\rangle$ the cyclic group of order a acting on its reflection representation $V=\mathbb{C}$ by $c \mapsto \zeta_{a}^{-1}$, and for any $s \in \mathbb{Z}$ (not necessarily coprime to a), the $V^{\otimes s}$-exponent of $G$ is $e_{1}^{G}\left(V^{\otimes s}\right)=a\left\lceil\frac{s}{a}\right\rceil-s$.

Proof. Note that $a\left\lceil\frac{s}{a}\right\rceil-s \in\{0, \ldots, a-1\}$ is congruent to $-s(\bmod a)$. Letting $x^{s}$ denote a basis vector for $\left(V^{\otimes s}\right)^{*}$, we see that $c\left(x^{s}\right)=\zeta_{a}^{s} x^{s}$ and therefore $x^{a\left\lceil\frac{s}{a}\right\rceil-s} \otimes x^{s}$ is a basis vector for $\left(U_{V^{\otimes s}}^{G}\right)^{*}=\left(\mathcal{C}_{G} \otimes\left(V^{\otimes s}\right)^{*}\right)^{G}$, because $\mathcal{C}_{G}=\operatorname{span}_{\mathbb{C}}\left\{1, x, \ldots, x^{a-1}\right\}$ in this case. 
Suppose now that $a=d e$ and consider $N=\left\langle c^{e}\right\rangle \simeq C_{d}$, which is a normal reflection subgroup of $G$ with quotient $H=G / N \simeq C_{e}$. We denote by $\zeta_{d}:=\zeta_{a}^{e}$ and $\zeta_{e}:=\zeta_{a}^{d}$, so that $N$ acts on $V^{*}$ via $c^{e} \mapsto \zeta_{d}$ and $H=\langle c N\rangle$ acts on $E^{*}=\operatorname{span}_{\mathbb{C}}\left\{x^{d}\right\}$ via $(c N)\left(x^{d}\right)=\zeta_{e} x^{d}$.

Taking again $s \in \mathbb{N}$ coprime to $a$ and letting $\sigma \in \operatorname{Gal}\left(\mathbb{Q}\left(\zeta_{a}\right) / \mathbb{Q}\right)$ be given by $\sigma: \zeta_{a} \mapsto \zeta_{a}^{s}$, we have that $E^{\sigma}$ is the 1-dimensional representation of $G$ defined by $c \mapsto \zeta_{a}^{-d s}$ or, equivalently, the 1-dimensional representation of $H$ defined by $c N \mapsto \zeta_{e}^{-s}$. Therefore, by Lemma 7.1, $e_{1}^{G}\left(E^{\sigma}\right)=a\left\lceil\frac{d s}{a}\right\rceil-d s$ and $e_{1}^{H}\left(E^{\sigma}\right)=e\left\lceil\frac{s}{e}\right\rceil-s$. On the other hand, $\left(U_{\sigma}^{N}\right)^{*}=\operatorname{span}_{\mathbb{C}}\left\{x^{d\left\lceil\frac{s}{d}\right\rceil-s} \otimes x^{\sigma}\right\}$ by Lemma 7.1, where again $x$ and $x^{\sigma}$ denote basis vectors for $V^{*}$ and $\left(V^{\sigma}\right)^{*}$, respectively. Hence, the generator $c$ of $G$ that acts by $\zeta_{a}^{-1}$ on its reflection representation $V$ now acts by $\zeta_{a}^{-d\left\lceil\frac{s}{d}\right\rceil}$ on $U_{\sigma}^{N}$ and therefore another application of Lemma 7.1 yields

$$
e_{1}^{G}\left(U_{\sigma}^{N}\right)=a\left\lceil\frac{\left\lceil\frac{s}{d}\right\rceil}{e}\right\rceil-d\left\lceil\frac{s}{d}\right\rceil .
$$

When $s=1$, we see that $\zeta_{a}^{-d\left\lceil\frac{s}{d}\right\rceil}=\zeta_{e}^{-1}$, so that $U^{N} \simeq E$ is the reflection representation of $H=C_{e}$ defined by $c N \mapsto \zeta_{e}^{-1}$. But because $\left\lceil\frac{s}{d}\right\rceil$ is not necessarily coprime to $e$, even when $s \in\{1, \ldots, a-1\}$, it is possible for the action of $H$ on $U_{\sigma}^{N}$ to fail to be faithful; for example, when $s=a-1$ corresponding to $\sigma$ acting by complex conjugation, the generator $c$ of $G$ acts trivially on $\left(U_{\sigma}^{N}\right)^{*}$ by $\zeta_{a}^{d\left\lceil\frac{a-1}{d}\right\rceil}=\zeta_{e}^{e}=1$. Hence, we see that, as we mentioned in Remark 4.4 and contrary to what one might have hoped based on the $s=1$ case, in general $U_{\sigma}^{N} \nsucceq E^{\sigma}$ as $G$-representations.

Using the explicit descriptions of the actions of $N$ and $G$ on $V^{\sigma}$ and the actions of $G$ and $H$ on $U_{\sigma}^{N}$ and $E^{\sigma}$ described above, the three equalities in Theorem 1.3 become the following numerological statements.

Corollary 7.2. Let $a=$ de and $s \in \mathbb{N}$ be coprime to $a$. Then

$$
\begin{aligned}
\left(d\left\lceil\frac{s}{d}\right\rceil-s\right)+\left(a\left\lceil\frac{\left\lceil\frac{s}{d}\right\rceil}{e}\right\rceil-d\left\lceil\frac{s}{d}\right\rceil\right) & =\left(a\left\lceil\frac{s}{a}\right\rceil-s\right) \\
d \cdot\left(e\left\lceil\frac{s}{e}\right\rceil-s\right) & =\left(a\left\lceil\frac{d s}{a}\right\rceil-d s\right) ; \\
d \cdot e & =a .
\end{aligned}
$$

The first (and only nontrivial) equality in this case is equivalent to the identity $\left\lceil\frac{\left\lceil\frac{s}{d}\right\rceil}{e}\right\rceil=$ $\left\lceil\frac{s}{d e}\right\rceil$, which holds more generally for $e \in \mathbb{N}$ and $s, d \in \mathbb{R}$.

Our Theorem 1.4 in this situation states that the following expressions are equal:

$$
\begin{aligned}
\sum_{g \in G}\left(\prod_{\lambda_{1}(g) \neq 1} \frac{1-\lambda_{1}(g)^{s}}{1-\lambda_{1}(g)}\right) q^{\mathrm{fix}_{V}(g)} t^{\mathrm{fix}_{E}(g)} & =q t+\left(\sum_{j=1}^{d-1} \frac{1-\zeta_{d}^{j s}}{1-\zeta_{d}^{j}}\right) t+\left(\sum_{\substack{a=1 \\
e \nmid k}}^{a-1} \frac{1-\zeta_{a}^{k s}}{1-\zeta_{a}^{k}}\right) \\
\left(q t+e_{1}^{N}\left(V^{\sigma}\right) t+e_{1}^{G}\left(U_{\sigma}^{N}\right)\right) & =q t+\left(d\left\lceil\frac{s}{d}\right\rceil-s\right) t+\left(a\left\lceil\frac{\left\lceil\frac{s}{d}\right\rceil}{e}\right\rceil-d\left\lceil\frac{s}{d}\right\rceil\right) .
\end{aligned}
$$


The equality of the coefficients of $t$

$$
\sum_{j=1}^{d-1} \frac{1-\zeta_{d}^{j s}}{1-\zeta_{d}^{j}}=d\left\lceil\frac{s}{d}\right\rceil-s=e_{1}^{N}\left(V^{\sigma}\right)
$$

was already verified above with $a$ in place of $d$. To verify the equality of constant terms

$$
\sum_{\substack{k=1 \\ e \nmid k}}^{a-1} \frac{1-\zeta_{a}^{k s}}{1-\zeta_{a}^{k}}=a\left\lceil\frac{\left\lceil\frac{s}{d}\right\rceil}{e}\right\rceil-d\left\lceil\frac{s}{d}\right\rceil
$$

one could proceed, for example, by noting that the same arguments show that the lefthand side is equal to

$$
\left(\sum_{k=1}^{a-1} \frac{1-\zeta_{a}^{k s}}{1-\zeta_{a}^{k}}\right)-\left(\sum_{j=1}^{d-1} \frac{1-\zeta_{d}^{j s}}{1-\zeta_{d}^{j}}\right)=\left(a\left\lceil\frac{s}{a}\right\rceil-s\right)-\left(d\left\lceil\frac{s}{d}\right\rceil-s\right)=a\left\lceil\frac{s}{a}\right\rceil-d\left\lceil\frac{s}{d}\right\rceil
$$

and then appealing either to Corollary 7.2 or to more general properties of ceilings to obtain the equality $\left\lceil\frac{\left\lceil\frac{s}{d}\right\rceil}{e}\right\rceil=\left\lceil\frac{s}{a}\right\rceil$.

We conclude our discussion of the cyclic case with a concrete illustration of the subtlety involved in proving (in Corollary 4.11) that the sum side of Theorem 1.4 provides the correct contribution coset by coset but not term by term as in the proof of Theorem 1.1.

To compare the two situations, we compute the trace of $n g=c^{e k} \cdot c^{j} \in N g$ (for $0 \leq k<d$ and $0 \leq j<e)$ acting on $S\left(V^{*}\right) \otimes \bigwedge\left(V^{\sigma}\right)^{*}$ and $S\left(V^{*}\right) \otimes \bigwedge\left(U_{\sigma}^{N}\right)^{*}$ :

$$
\begin{aligned}
& \sum_{\ell, p \geq 0} \operatorname{tr}\left((n g) \mid S\left(V^{*}\right)_{\ell} \otimes \bigwedge^{p}\left(V^{\sigma}\right)^{*}\right) x^{\ell} u^{p}=\left.\frac{1+\left.u\left(c^{e k+j}\right)\right|_{\left(V^{\sigma}\right)^{*}}}{1-\left.x\left(c^{e+j}\right)\right|_{(V)^{*}}}\right|_{\substack{u=q(1-x)-1 \\
x \rightarrow 1}} \\
& =\left.\frac{1+u \zeta_{a}^{s(e k+j)}}{1-x \zeta_{a}^{e k+j}}\right|_{\substack{u=q(1-x)-1 \\
x \rightarrow 1}} \\
& =\left.\left(\frac{1-\zeta_{a}^{s(e k+j)}}{1-x \zeta_{a}^{e k+j}}+\frac{q(1-x) \zeta_{a}^{s(e k+j)}}{1-x \zeta_{a}^{e k+j}}\right)\right|_{x \rightarrow 1} \\
& =\left(\prod_{\lambda_{1}(n g) \neq 1} \frac{1-\lambda_{1}(n g)^{\sigma}}{1-\lambda_{1}(n g)}\right) q^{\mathrm{fix}_{V}(n g)}
\end{aligned}
$$

because the term $\frac{q(1-x) \zeta_{a}^{s(e k+j)}}{1-x \zeta_{a}^{e k+j}}$ vanishes in the limit $x \rightarrow 1$ for all elements of $G$ except the identity. For example, for $a=6, d=2, e=3$ and $s=5$, summing over all elements of 
$C_{6}=\langle c\rangle$ and then specialising $u \mapsto q(1-x)-1$ and taking the limit as $x \rightarrow 1$ gives

\begin{tabular}{c|cc|cc}
\multicolumn{2}{c}{$N$} & \multicolumn{2}{c}{$c N$} & \multicolumn{2}{c}{$c^{2} N$} \\
$\mathrm{id} \quad c^{3}$ & \multicolumn{1}{c|}{$c$} & $c^{4}$ & $c^{2}$ & $c^{5}$ \\
\hline$q+\frac{1-\zeta_{6}^{3}}{1-\zeta_{6}^{3}}$ & $\frac{1-\zeta_{6}^{5}}{1-\zeta_{6}}+\frac{1-\zeta_{6}^{2}}{1-\zeta_{6}^{4}}$ & $\frac{1-\zeta_{6}^{4}}{1-\zeta_{6}^{2}}+\frac{1-\zeta_{6}^{1}}{1-\zeta_{6}^{5}}$ & 0
\end{tabular}

so that each element contributes the 'correct amount' specified by the sum side of Theorem 1.1.

On the other hand, computing the sum side of our refinement Theorem 1.4 gives the following, where we have written $b:=e_{1}^{N}\left(V^{\sigma}\right)=d\left\lceil\frac{s}{d}\right\rceil-s$ for legibility:

$$
\begin{aligned}
& \sum_{\ell, p \geq 0} \operatorname{tr}\left((n g) \mid S\left(V^{*}\right)_{\ell} \otimes \bigwedge^{p}\left(U_{\sigma}^{N}\right)^{*}\right) x^{\ell} y^{b p} u^{p}=\left.\frac{1+\left.u y^{b}\left(c^{e k+j}\right)\right|_{\left(U_{\sigma}^{N}\right)_{b}^{*}}}{1-\left.x\left(c^{e k+j}\right)\right|_{(V)^{*}}}\right|_{\substack{u=q t(1-x)-1 \\
x \rightarrow 1}} \\
& =\left.\frac{1+u x^{t b} \zeta_{e}^{\left\lceil\frac{s}{d}\right\rceil(e k+j)}}{1-x \zeta_{a}^{e k+j}}\right|_{\substack{u=q t(1-x)-1 \\
x \rightarrow 1}} \\
& =\left.\left(\frac{1-x^{t b} \zeta_{e}^{j\left\lceil\frac{s}{d}\right\rceil}}{1-x \zeta_{a}^{e k+j}}+\frac{x^{t b} q t(1-x) \zeta_{e}^{j\left\lceil\frac{s}{d}\right\rceil}}{1-x \zeta_{a}^{e k+j}}\right)\right|_{x \rightarrow 1} \\
& = \begin{cases}q t+b t & \text { if } k=j=0 \\
\frac{1-\zeta_{e}^{j\left\lceil\frac{s}{d}\right\rceil}}{1-\zeta_{a}^{e k+j}} & \text { otherwise. }\end{cases}
\end{aligned}
$$

Continuing the example with $a=6, d=2, e=3$ and $s=5$, because $\left\lceil\frac{s}{d}\right\rceil=e=3$, after specialising $y \mapsto x^{t}, u \mapsto q t(1-x)-1$, and taking the limit as $x \rightarrow 1$, every term except for the identity vanishes - in particular, each element of $G$ does not contribute the 'correct amount' specified by the sum side of Theorem 1.4:

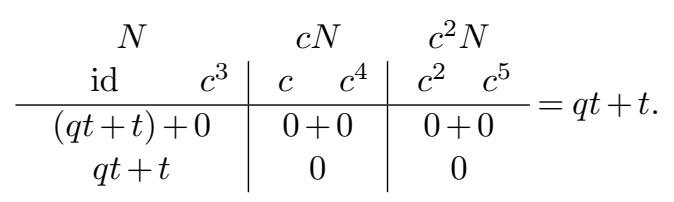

Here the only coset that provides a nontrivial contribution to the sum side of Theorem 1.4 is the trivial coset $N$, as predicted by the computation of the sum side of Theorem 1.1, but this nontrivial contribution of $q t+t$ for the whole coset $N$ is concentrated on the identity element $c^{0} \in N$ alone, which, according to the sum side of Theorem 1.4, should have only contributed $q t$, whereas the nontrivial element $c^{3} \in N$ did not provide the correct contribution of $\frac{1-\lambda_{1}\left(c^{3}\right)^{\sigma}}{1-\lambda_{1}\left(c^{3}\right)} q^{\mathrm{fix}_{V}\left(c^{3}\right)} t^{\mathrm{fix}_{E}\left(c^{3}\right)}=t$ specified by the sum side of Theorem 1.4.

\subsection{The Infinite Family $G(a b, b, r)$}

We begin by defining an ad hoc operation on $G$-modules for $G=G(a b, b, r)$ (whose definition was given in Section 6) that will allow us to succintly identify the Orlik-Solomon 
spaces $U_{\sigma}^{N}$ of Definition 2.9 for the two kinds of normal reflection subgroups $N$ of $G$ listed in Theorem 6.1 that occur in all ranks $r \geq 2$.

Definition 7.3. For $n \in \mathbb{Z}$, let $\mu_{n}: G \rightarrow G$ be the group endomorphism obtained by raising each nonzero matrix entry to the $n$th power. Given any representation $\rho: G \rightarrow \operatorname{GL}(W)$, we define the fake tensor power $W^{\bigotimes n}$ as the representation $\rho \circ \mu_{n}: G \rightarrow \operatorname{GL}(W)$.

Remark 7.4. Note that in general the 'fake $n$th power map' $\mu_{n}$ used in Definition 7.3 will be neither injective nor surjective when $\operatorname{gcd}(a b, n) \neq 1$. In the case where $\operatorname{gcd}(a b, s)=1$, note that $V^{\bigotimes s} \simeq V^{\sigma}$, the Galois twist corresponding to $\sigma: \zeta_{a b} \mapsto \zeta_{a b}^{s}$.

Although the fake tensor power operation could be expressed in terms of more systematic constructions (interpreting $G(a b, b, r)$ as an index- $b$ subgroup of the wreath product $C_{a b}\left(S_{r}\right)$, we have preferred our ad hoc definition for its simplicity and concreteness.

In the following result, we identify the $G$-module $U_{\sigma}^{N}$ when $N=C_{d}^{r}$ as in Theorem $6.1(r . a)$ for $r \geq 2$.

Proposition 7.5. Let $a=$ de and $N=C_{d}^{r} \triangleleft G(a b, b, r)=G$ and fix $\sigma: \zeta_{a b} \rightarrow \zeta_{a b}^{s}$ for $1 \leq$ $s<a b$ with $\operatorname{gcd}(s, a b)=1$. Then $H \simeq G(e b, b, r)$ and $U_{\sigma}^{N} \simeq E^{\otimes\left\lceil\frac{s}{d}\right\rceil}$ as G-modules.

Proof. For this choice of $N$, the fundamental $N$-invariants are $N_{i}=x_{i}^{d}$ for $1 \leq i \leq r$, and we obtain the basis $u_{i}^{N}=x_{i}^{d\left\lceil\frac{s}{d}\right\rceil-s} \otimes x_{i}^{\sigma}$ for $\left(U_{\sigma}^{N}\right)^{*}$ as in Section 7.1.

Fix $g \in G$ and $\ell \in\{1, \ldots, r\}$ and suppose that $g\left(x_{\ell}\right)=\zeta_{a b}^{k} x_{j}$ for some $0 \leq k<a b$ and $j \in\{1, \ldots, r\}$. Then from the explicit descriptions of $E^{*}$ and $\left(U_{\sigma}^{N}\right)^{*}$ above we see that $g\left(N_{\ell}\right)=\zeta_{a b}^{k d} N_{j}$ and $g\left(u_{\ell}^{N}\right)=\zeta_{a b}^{k d\left\lceil\frac{s}{d}\right\rceil} u_{j}^{N}$. It follows that $E \simeq V^{\bigotimes d}$ and $U_{\sigma}^{N} \simeq V^{\bigotimes d\left\lceil\frac{s}{d}\right\rceil}$, and therefore $U_{\sigma}^{N} \simeq E^{\otimes\left\lceil\frac{s}{d}\right\rceil}$, as claimed.

As a special case of Proposition 7.5, $U_{\sigma}^{N} \simeq E$ when $N=C_{d}^{r}$ and $s<d$.

Following [18, Proposition 14.1], a simple choice of invariant polynomials for $G(a b, b, r)$ is the set

$$
G_{i}=\sum_{j=1}^{r} x_{j}^{a b i} \quad \text { for } 1 \leq i<r \quad \text { and } \quad G_{r}=\left(x_{1} \cdots x_{r}\right)^{a} .
$$

In the following result we determine the Orlik-Solomon space $U_{\sigma}^{G}$ of Definition 2.9 up to graded cryptomorphism.

Theorem 7.6. Let $G=G(a b, b, r)$ and $\sigma \in \operatorname{Gal}\left(\mathbb{Q}\left(\zeta_{a b}\right) / \mathbb{Q}\right)$ be given by $\sigma\left(\zeta_{a b}\right)=\zeta_{a b}^{s}$ for $1 \leq s<a b$ with $\operatorname{gcd}(s, a b)=1$. Let $\left(\tilde{U}_{\sigma}^{G}\right)^{*}:=\operatorname{span}_{\mathbb{C}}\left\{\tilde{u}_{1}^{G}, \ldots, \tilde{u}_{r}^{G}\right\}$, where

$$
\tilde{u}_{i}^{G}=\sum_{j=1}^{r} x_{j}^{a b i-s} \otimes x_{j}^{\sigma} \quad \text { for } \quad 1 \leq i<r \quad \text { and } \quad \tilde{u}_{r}^{G}=\sum_{j=1}^{r}\left(x_{1} \cdots x_{r}\right)^{\left\lceil\frac{s}{a}\right\rceil a} x_{j}^{-s} \otimes x_{j}^{\sigma} .
$$

Let $\eta_{G}: S\left(V^{*}\right) \rightarrow \mathcal{C}_{G}$ denote the natural projection from $S\left(V^{*}\right)$ onto its $G$-stable direct summand $\mathcal{C}_{G}$. Then $\eta_{G} \otimes 1:\left(\tilde{U}_{\sigma}^{G}\right)^{*} \rightarrow\left(U_{\sigma}^{G}\right)^{*}$ is an isomorphism of graded vector spaces, 
and therefore the $V^{\sigma}$-exponents of $G(a b, b, r)$ are

$$
\left\{a b-s, 2 a b-s, \ldots,(r-1) a b-s,\left\lceil\frac{s}{a}\right\rceil a r-s\right\} .
$$

Proof. By Lemma 3.6 (applied in the special case where $N=G$ ), it suffices to show that the $\tilde{u}_{i}^{G}$ form a basis for $\left(S\left(V^{*}\right) \otimes\left(V^{\sigma}\right)^{*}\right)^{G}$ as a free $S\left(V^{*}\right)^{G}$-module.

Because the group $G(a b, b, r)$ acts on $V^{*}$ by permutation of the $x_{i}$ and multiplication by (ab)th roots of unity, it is clear that the $\tilde{u}_{i}^{G} \in S\left(V^{*}\right) \otimes\left(V^{\sigma}\right)^{*}$ are $G$-invariant. Let $u_{i}^{G}=$ $\sum_{j=0}^{r} a_{i j}^{G} \otimes x_{j}^{\sigma}$ be a homogeneous basis for $\left(U_{\sigma}^{G}\right)^{*}$, where $a_{i j}^{G} \in \mathcal{C}_{G}$ and $\operatorname{deg}\left(a_{i j}^{G}\right)=e_{i}^{G}\left(V^{\sigma}\right)$ whenever $a_{i j}^{G} \neq 0$. Because $\left(S\left(V^{*}\right) \otimes\left(V^{\sigma}\right)^{*}\right)^{G} \simeq S\left(V^{*}\right)^{G} \otimes\left(U_{\sigma}^{G}\right)^{*}$, there exists a matrix $\left[p_{i j}^{G}\right] \in \operatorname{Mat}_{r \times r}\left(S\left(V^{*}\right)^{G}\right)$ such that $\left[p_{i j}^{G}\right] \cdot\left[a_{i j}^{G}\right]=\left[\tilde{a}_{i j}^{G}\right]$, where

$$
\tilde{a}_{i j}^{G}= \begin{cases}x_{j}^{a b i-s} & \text { for } 1 \leq i<r \\ \left(x_{1} \cdots x_{r}\right)^{\left\lceil\frac{s}{a}\right\rceil a} x_{j}^{-s} & \text { for } i=r\end{cases}
$$

or, equivalently, such that $\tilde{u}_{i}^{G}=\sum_{j=1}^{r} p_{i j}^{G} \cdot u_{j}^{G}$. It is clear from the form of the $\tilde{u}_{i}^{G}$ that they are $\mathbb{C}$-linearly independent, which implies that $\operatorname{det}\left(p_{i j}^{G}\right) \neq 0$.

We claim that

$$
\operatorname{det}\left(a_{i j}^{G}\right)=c \cdot\left(x_{1} \cdots x_{r}\right)^{\left\lceil\frac{s}{a}\right\rceil a-s} \prod_{1 \leq i<j \leq r}\left(x_{i}^{a b}-x_{j}^{a b}\right)
$$

for some $0 \neq c \in \mathbb{C}$. By Gutkin's theorem [14, Theorem 10.13],

$$
\operatorname{det}\left(a_{i j}^{G}\right)=c \cdot \prod_{H \in \mathcal{R}_{G}} L_{H}^{C\left(H, V^{\sigma}\right)}
$$

for some $0 \neq c \in \mathbb{C}$, where $\mathcal{R}_{G}$ denotes the set of reflecting hyperplanes for $G, L_{H} \in$ $V^{*}$ denotes a linear form defining $H$ and $C\left(H, V^{\sigma}\right)$ is defined as follows. Denoting by $\left\langle r_{H}\right\rangle=G_{H}<G$ the cyclic subgroup of $G$ that stabilises $H$ pointwise, decompose $\left(V^{\sigma}\right)^{*} \simeq$ $\bigoplus_{i=1}^{r} \lambda^{\otimes k_{i}}$ as a $G_{H}$-module with $0 \leq k_{i}<\left|G_{H}\right|$ for $1 \leq i \leq r$, where $\lambda$ denotes the standard representation of $G_{H}$ on the $G_{H}$-stable complement of $H$ in $V$, and define $C\left(H, V^{\sigma}\right):=$ $\sum_{i=1}^{r} k_{i}$. When $L_{H}=x_{i}-\zeta_{a b}^{\ell} x_{j}$ with $i \neq j$ and $0 \leq \ell<a b-1$, the cyclic generator $r_{H}$ has order 2 and $V^{\sigma} \simeq \lambda \oplus \mathbb{C}^{\oplus(r-1)}$ as a $G_{H}$-module, and therefore $C\left(H, V^{\sigma}\right)=1$ in this case. If $a>1$, then we have the additional reflecting hyperplanes defined by $L_{H}=x_{i}$; in this case, the cyclic generator $r_{H}$ has order $a$ and $\left(V^{\sigma}\right)^{*} \simeq \lambda^{\otimes\left(\left\lceil\frac{s}{a}\right\rceil a-s\right)} \oplus \mathbb{C}^{\oplus(r-1)}$ as a $G_{H}$-module (cf. Lemma 7.1), and therefore $C\left(H, V^{\sigma}\right)=\left\lceil\frac{s}{a}\right\rceil a-s$. This concludes the proof of Equation (15).

We see by direct inspection that

$$
\operatorname{deg}\left(\operatorname{det}\left(a_{i j}^{G}\right)\right)=a b \cdot\left(\begin{array}{l}
r \\
2
\end{array}\right)+r \cdot\left(\left\lceil\frac{s}{a}\right\rceil a-s\right)=\operatorname{deg}\left(\operatorname{det}\left(\tilde{a}_{i j}^{G}\right)\right),
$$

which implies that $\operatorname{deg}\left(\operatorname{det}\left(p_{i j}^{G}\right)\right)=0$, and because $\operatorname{det}\left(p_{i j}^{G}\right) \neq 0$ as we had already seen, it follows that $\left[p_{i j}^{G}\right] \in \operatorname{GL}_{r}\left(S\left(V^{*}\right)^{G}\right)$, as we wanted to show.

Remark 7.7. Note that when $s=a b-1$, so that $\sigma$ acts by complex conjugation, the basis $\tilde{u}_{i}{ }^{G}$ for $\left(\tilde{U}_{\sigma}^{G}\right)^{*}$ in Theorem 7.6 agrees with the one computed in [16, Section 6$]$. 
In the following result, we identify the $G$-module $U_{\sigma}^{N}$ when $N=G(a b, d b, r)$ as in Theorem $6.1(r . \mathrm{b})$ for $r \geq 2$.

Proposition 7.8. Let $a=$ de and $N=G(a b, d b, r) \unlhd G(a b, b, r)=G$, and fix $\sigma: \zeta_{a b} \rightarrow \zeta_{a b}^{s}$ for $1 \leq s<a b$ with $\operatorname{gcd}(s, a b)=1$. Then $H \simeq C_{d}$ and $U_{\sigma}^{N} \simeq E^{\otimes\left\lceil\frac{s}{e}\right\rceil}$ as G-modules.

Proof. Note that for this choice of $G$ and $N$, with fundamental invariants defined as in Equation (14), we have $N_{i}=G_{i}$ for $i=1, \ldots, r-1$, and $N_{r}=\left(x_{1} \cdots x_{r}\right)^{e}$ while $G_{r}=$ $\left(x_{1} \cdots x_{r}\right)^{a}$. The matrix $\operatorname{diag}\left(\zeta_{a}, 1, \ldots, 1\right) \in G$ maps to the cyclic generator $c \in C_{d} \simeq H$, acting trivially on $\operatorname{span}_{\mathbb{C}}\left\{N_{1}, \ldots, N_{r-1}\right\}$ and mapping $c: N_{r} \mapsto \zeta_{d} N_{r}$, where $\zeta_{d}:=\zeta_{a}^{-e}$ is a primitive $d$ th root of unity.

On the other hand, we see that the space $\left(\tilde{U}_{\sigma}^{N}\right)^{*}=\operatorname{span}\left\{\tilde{u}_{1}^{N}, \ldots, \tilde{u}_{r}^{N}\right\}$ constructed in Theorem 7.6 is $G$-stable and the $\tilde{u}_{i}^{N}$ form a homogeneous basis for $\left(S\left(V^{*}\right) \otimes\left(V^{\sigma}\right)^{*}\right)^{N}$ as a free $S\left(V^{*}\right)^{N}$-module, and therefore it is isomorphic to $\left(U_{\sigma}^{N}\right)^{*}$ as a graded $G$-module by Lemma 3.6. We see that $\operatorname{diag}\left(\zeta_{a}, 1, \ldots, 1\right) \in G$ acts trivially on $\operatorname{span}_{\mathbb{C}}\left\{\tilde{u}_{1}^{N}, \ldots, \tilde{u}_{r-1}^{N}\right\}$ and $\operatorname{maps} c: \tilde{u}_{r}^{N} \mapsto \zeta_{a}^{-e\left\lceil\frac{s}{e}\right\rceil} \tilde{u}_{r}^{N}=\zeta_{d}^{\left\lceil\frac{s}{e}\right\rceil} \tilde{u}_{r}^{N}$.

Note that we do not necessarily have that $\left\lceil\frac{s}{e}\right\rceil$ is relatively prime to $d$. When $d=a$ so that $e=1$ and $N=G(a b, a b, r), G / N \simeq C_{a}=\langle c\rangle$ acts by $c: u_{r}^{N} \rightarrow \zeta_{a}^{-s} u_{r}^{N}$, so that $U_{\sigma}^{N} \simeq E^{\sigma}$. Another special case occurs when $s<e$, so that $U_{\sigma}^{N} \simeq E$.

In the following result, we identify the representation $U_{\sigma}^{N}$ when $G=(2 a b, 2 d, 2)$ and $N=G(a b, a, 2)$ as in Theorem 6.1(2.c). In this case it is not possible to write $U_{\sigma}^{N}$ as a fake tensor power (Definition 7.3) of $E$ in general.

Proposition 7.9. Let $a=$ de and $N=G(a b, a, 2) \triangleleft G(2 a b, 2 d, 2)=G$, and fix $\sigma: \zeta_{2 a b} \mapsto \zeta_{2 a b}^{s}$ for $1 \leq s<2 a b$ with $\operatorname{gcd}(s, 2 a b)=1$. Then $H \simeq C_{2} \times C_{e}$ and $E \simeq \lambda_{2} \oplus \lambda_{e}$, where $\lambda_{2}$ is the standard reflection representation of $C_{2}$ and $\lambda_{e}$ is the standard reflection representation of $C_{e}$, and $U_{\sigma}^{N} \simeq \lambda_{2}^{\otimes\left\lceil\frac{s}{a b}\right\rceil} \oplus \lambda_{e}^{\otimes\left\lceil\frac{s}{b}\right\rceil}$ as H-modules.

Proof. We may choose the fundamental $N$-invariants

$$
N_{1}(\mathbf{x})=x_{1}^{a b}+x_{2}^{a b} \quad \text { and } \quad N_{2}(\mathbf{x})=\left(x_{1} x_{2}\right)^{b}
$$

as in Equation (14). Letting

$$
h_{1}:=\left(\begin{array}{cc}
0 & \zeta_{2 a b} \\
\zeta_{2 a b}^{-1} & 0
\end{array}\right) \quad \text { and } \quad h_{2}:=\left(\begin{array}{cc}
\zeta_{e b} & 0 \\
0 & 1
\end{array}\right) .
$$

We see that $h_{i}\left(N_{j}\right)=N_{j}$ if $i \neq j, h_{1}\left(N_{1}\right)=-N_{1}$, and $h_{2}\left(N_{2}\right)=\zeta_{e} N_{2}$, where $\zeta_{e}:=\zeta_{e b}^{-b}$, and therefore $h_{1}$ and $h_{2}$ map to the generators of the cyclic factors in the bicyclic quotient group $H \simeq C_{2} \times C_{e}$. By Theorem 7.6 and Lemma $3.6\left(\tilde{U}_{\sigma}^{N}\right)^{*}=\operatorname{span}\left\{\tilde{u}_{1}^{N}, \tilde{u}_{2}^{N}\right\}$ is isomorphic to $\left(U_{\sigma}^{N}\right)^{*}$ as a graded $G$-module, where

$$
\begin{aligned}
& \tilde{u}_{1}^{N}=x_{1}^{\left\lceil\frac{s}{a b}\right\rceil a b-s} \otimes x_{1}^{\sigma}+x_{2}^{\left\lceil\frac{s}{a b}\right\rceil a b-s} \otimes x_{2}^{\sigma} ; \quad \text { and } \\
& \tilde{u}_{2}^{N}=\left(x_{1} x_{2}\right)^{\left\lceil\frac{s}{b}\right\rceil b} x_{1}^{-s} \otimes x_{1}^{\sigma}+\left(x_{1} x_{2}\right)^{\left\lceil\frac{s}{b}\right\rceil b} x_{2}^{-s} \otimes x_{2}^{\sigma} .
\end{aligned}
$$

We see that $h_{i}\left(\tilde{u}_{j}^{N}\right)=\tilde{u}_{j}^{N}$ if $i \neq j, h_{1}\left(\tilde{u}_{1}^{N}\right)=(-1)^{\left\lceil\frac{s}{a b}\right\rceil} \tilde{u}_{1}^{N}$, and $h_{2}\left(\tilde{u}_{2}^{N}\right)=\zeta_{e}^{\left\lceil\frac{s}{b}\right\rceil} \tilde{u}_{2}^{N}$. 
As before, note that we do not necessarily have that $\left\lceil\frac{s}{b}\right\rceil$ is relatively prime to $e$. When $b=1$, we have that $U_{\sigma}^{N} \simeq E^{\sigma}$ whenever $s<a$. When $a=1$ we do have $U_{\sigma}^{N} \simeq E^{\otimes\left\lceil\frac{s}{b}\right\rceil}$ as in the previous cases. Another special case occurs when $s<b$, so that $U_{\sigma}^{N} \simeq E$.

\subsection{An exceptional example $G_{15} \triangleright G_{12}$}

To illustrate the choice of indexing of degrees and exponents that is needed in Theorem 1.3, consider

$$
G:=G_{15}=\left\langle\left(\begin{array}{cc}
1 & 0 \\
0 & -1
\end{array}\right), \frac{\zeta_{3}}{2}\left(\begin{array}{cc}
-1-i & 1-i \\
-1-i & -1+i
\end{array}\right), \frac{1}{\sqrt{2}}\left(\begin{array}{cc}
1 & -1 \\
-1 & -1
\end{array}\right)\right\rangle
$$

where we use the conventions in [14, Chapter 3] and the given matrices describe the action of $G$ on $V^{*}$. We will write the reflection generators of this non-well-generated reflection group $G$ as $\mathbf{s}, \mathbf{t}$ and $\mathbf{u}$, in the order they appear above. The degrees of $G$ are $d_{1}^{G}=12$ and $d_{2}^{G}=24$, with corresponding invariant generators of $S\left(V^{*}\right)^{G}$ given by

$$
G_{1}(x, y)=\left(x^{5} y-x y^{5}\right)^{2} \quad \text { and } \quad G_{2}(x, y)=\left(x^{8}+14 x^{4} y^{4}+y^{8}\right)^{3} .
$$

We then have the following normal reflection subgroup $N \unlhd G$ generated by the reflections $\mathbf{u}$, sus $^{-1}$ and $\mathbf{t s u s}^{-1} \mathbf{t}^{-1}$ :

$$
N:=G_{12}=\left\langle\frac{1}{\sqrt{2}}\left(\begin{array}{cc}
1 & -1 \\
-1 & -1
\end{array}\right), \frac{1}{\sqrt{2}}\left(\begin{array}{cc}
1 & 1 \\
1 & -1
\end{array}\right),\left(\begin{array}{cc}
0 & \zeta_{8} \\
\zeta_{8}^{-1} & 0
\end{array}\right)\right\rangle,
$$

where again the above matrices describe the acion of $N$ on its dual reflection representation $V^{*}$. The degrees of $N$ are $d_{1}^{N}=6$ and $d_{2}^{N}=8$, with corresponding invariant generators of $S\left(V^{*}\right)^{N}$ given by

$$
N_{1}(x, y)=x^{5} y-x y^{5} \quad \text { and } \quad N_{2}(x, y)=x^{8}+14 x^{4} y^{4}+y^{8} .
$$

Then the quotient group $H:=G / N \simeq C_{2} \times C_{3}$, and by Theorem $1.2 \mathrm{H}$ acts in a reflection representation on $E$, where $E^{*}=\operatorname{span}_{\mathbb{C}}\left\{N_{1}, N_{2}\right\}$, because

$$
\begin{array}{ccr}
\mathbf{s}\left(N_{1}\right)=-N_{1}, & \mathbf{s}\left(N_{2}\right)=N_{2} \\
\mathbf{t}\left(N_{1}\right)=N_{1}, & \mathbf{t}\left(N_{2}\right)=\zeta_{3}^{2} N_{2} \\
\mathbf{u}\left(N_{1}\right)=N_{1}, & \mathbf{u}\left(N_{2}\right)=N_{2} .
\end{array}
$$

Compatible with the choices of invariant generators $G_{1}$ and $G_{2}$ for $G$ and $N_{1}$ and $N_{2}$ for $N$, we find invariant generators of $S\left(E^{*}\right)^{H}$ that are $\mathbf{N}$-homogeneous of degrees $d_{1}^{H}=2$ and $d_{2}^{H}=3$ :

$$
H_{1}\left(N_{1}, N_{2}\right)=N_{1}^{2}=G_{1}(x, y) \quad \text { and } \quad H_{2}\left(N_{1}, N_{2}\right)=N_{2}^{3}=G_{2}(x, y),
$$

and also $\mathbf{x}$-homogeneous of degrees $d_{1}^{G}=12=d_{1}^{N} \cdot d_{1}^{H}$ and $d_{2}^{G}=24=d_{2}^{N} \cdot d_{2}^{H}$.

As we can see from the explicit matrices above, the reflection representation of $G$ is actually defined over $\zeta_{24}$. For $s$ coprime to 24 we write $\sigma_{s}$ for the Galois automorphism $\sigma_{s} \in \operatorname{Gal}\left(\mathbb{Q}\left(\zeta_{24}\right) / \mathbb{Q}\right)$ defined by $\sigma_{s}: \zeta_{24} \mapsto \zeta_{24}^{s}$. The complete data for every Galois twist $V^{\sigma}$ for $G=G_{15}$ and $N=G_{12}$ appear in Table 2. Code for computing similar examples using Sage can be found at [2]. 
TABle 2. Data for $G=G_{15}, N=G_{12}$ and $H=G / N=C_{2} \times C_{3}$ computed using [2]. The rows are indexed by the Galois twists $\sigma_{s}: \zeta_{24} \rightarrow \zeta_{24}^{s}$ (for $s$ coprime to 24). The columns contain the degrees of $N$ multiplied by the $E^{\sigma}$-exponents of $H$ to obtain the $E^{\sigma}$-exponents of $G$ and the $V^{\sigma}$-exponents of $N$ added to the $U_{\sigma}^{N}$-exponents of $G$ to obtain the $V^{\sigma}$-exponents of $G$, indexed according to Theorem 1.3. The final column lists the corresponding product sides of the weighted sums over $G$ according to Theorem 1.4.

\begin{tabular}{|c|ccc|ccc|c|}
\hline$s$ & \multicolumn{2}{|l|}{$d_{i}^{N} \cdot e_{i}^{H}\left(E^{\sigma}\right)=e_{i}^{G}\left(E^{\sigma}\right)$} & \multicolumn{2}{|c|}{$e_{i}^{N}\left(V^{\sigma}\right)+e_{i}^{G}\left(U_{\sigma}^{N}\right)=e_{i}^{G}\left(V^{\sigma}\right)$} & product side \\
\hline 1 & 6,8 & 1,2 & 6,16 & 5,7 & 6,16 & 11,23 & $(q t+5 t+6)(q t+7 t+16)$ \\
5 & 6,8 & 1,1 & 6,8 & 1,11 & 6,8 & 7,19 & $(q t+t+6)(q t+11 t+8)$ \\
7 & 6,8 & 1,2 & 6,16 & 11,1 & 6,16 & 17,17 & $(q t+11 t+6)(q t+t+16)$ \\
11 & 6,8 & 1,1 & 6,8 & 7,5 & 6,8 & 13,13 & $(q t+7 t+6)(q t+5 t+8)$ \\
13 & 6,8 & 1,2 & 6,16 & 11,1 & 0,22 & 11,23 & $(q t+11 t)(q t+t+22)$ \\
17 & 6,8 & 1,1 & 6,8 & 7,5 & 0,14 & 7,19 & $(q t+7 t)(q t+5 t+14)$ \\
19 & 6,8 & 1,2 & 6,16 & 5,7 & 0,22 & 5,29 & $(q t+5 t)(q t+7 t+22)$ \\
23 & 6,8 & 1,1 & 6,8 & 1,11 & 0,14 & 1,25 & $(q t+t)(q t+11 t+14)$ \\
\hline
\end{tabular}

Let us work out the case $\sigma=\sigma_{13}$ in detail; the other cases are similar. Writing $x^{\sigma}$ and $y^{\sigma}$ for the basis vectors of $\left(V^{\sigma}\right)^{*}$ as before, we find bases $\left\{u_{1}^{G}, u_{2}^{G}\right\}$ for $\left(U_{\sigma}^{G}\right)^{*}$ and $\left\{u_{1}^{N}, u_{2}^{N}\right\}$ for $\left(U_{\sigma}^{N}\right)^{*}$ given by

$$
\begin{aligned}
u_{1}^{G} & =\left(x^{11}-22 x^{7} y^{4}-11 x^{3} y^{8}\right) \otimes x^{\sigma} \\
& +\left(y^{11}-22 x^{4} y^{7}-11 x^{8} y^{3}\right) \otimes y^{\sigma} \\
u_{2}^{G} & =\left(\left(x^{21} y^{2}-x y^{22}\right)+27\left(x^{17} y^{6}-x^{5} y^{18}\right)+170\left(x^{13} y^{10}-x^{9} y^{14}\right)\right) \otimes x^{\sigma} \\
& +\left(\left(x^{2} y^{21}-x^{22} y\right)+27\left(x^{6} y^{17}-x^{18} y^{5}\right)+170\left(x^{10} y^{13}-x^{14} y^{9}\right)\right) \otimes y^{\sigma} \\
u_{1}^{N} & =u_{1}^{G} ; \text { and } \\
u_{2}^{N} & =y \otimes x^{\sigma}-x \otimes y^{\sigma} .
\end{aligned}
$$

Writing $u_{i}^{G}=a_{i 1}^{G} \otimes x^{\sigma}+a_{i 2}^{G} \otimes y^{\sigma}$ and $u_{i}^{N}=a_{i 1}^{N} \otimes x^{\sigma}+a_{i 2}^{N} \otimes y_{\sigma}$ as above, let us verify that $a_{i j}^{G} \in \mathcal{C}_{G}$ and $a_{i j}^{N} \in \mathcal{C}_{N}$. It is clear that $a_{11}^{G}, a_{12}^{G} \in \mathcal{C}_{G}$, because $\operatorname{deg}\left(a_{1 j}^{G}\right)=11<d_{1}^{G}$, di $d_{2}^{G}$, and every polynomial of degree smaller than every degree of $G$ belongs to $\mathcal{C}_{G}$. Similarly, we see that $a_{21}^{N}, a_{22}^{N} \in \mathcal{C}_{N}$, because $\operatorname{deg}\left(a_{2 j}^{N}\right)=1<d_{1}^{N}, d_{2}^{N}$. We observe that $u_{2}^{G}=N_{1} N_{2}^{2} \cdot u_{2}^{N}$ (we discuss the meaning of this observation in more detail below), and therefore $u_{2}^{G} \in$ $\mathcal{C}_{G} \otimes\left(V^{\sigma}\right)^{*} \simeq \mathcal{C}_{H} \otimes \mathcal{C}_{N} \otimes\left(V^{\sigma}\right)^{*}$ by Proposition 3.3. To see that $u_{1}^{N} \in \mathcal{C}_{N} \otimes\left(V^{\sigma}\right)^{*}$ also, suppose that $w_{1}^{N}, u_{2}^{N}$ is a homogeneous basis for $\left(U_{\sigma}^{N}\right)^{*}$, and let $p_{1}, p_{2} \in S\left(V^{*}\right)^{N}$ be homogeneous such that $u_{1}^{N}=p_{1} w_{1}^{N}+p_{2} u_{2}^{N}$. But then $p_{2}=0$, because no homogeneous $N$ invariant polynomial has degree 10 , which implies that $a_{1 j}^{N}$ is divisible by a homogeneous $N$-invariant polynomial $p_{1}$ with $\operatorname{deg}\left(p_{1}\right) \leq 11$. But the only nonconstant choices are $p_{1}=N_{1}$ or $p_{1}=N_{2}$, none of which divide the coefficients $a_{1 j}^{N}$ above, and therefore $p_{1} \in \mathbb{C}^{\times}$. This concludes the proof that the $u_{i}^{G}$ and $u_{i}^{N}$ specified above are indeed bases for $\left(U_{\sigma}^{G}\right)^{*}$ and $\left(U_{\sigma}^{N}\right)^{*}$, respectively, as claimed. 
Hence, the $V^{\sigma}$-exponents of $G$ are $e_{1}^{G}\left(V^{\sigma}\right)=11$ and $e_{2}^{G}\left(V^{\sigma}\right)=23$, and the $V^{\sigma}$-exponents of $N$ are $e_{1}^{N}\left(V^{\sigma}\right)=11$ and $e_{2}^{N}\left(V^{\sigma}\right)=1$. We compute the action of $G$ on $\left(U_{\sigma}^{N}\right)^{*}$ and obtain

$$
\begin{aligned}
& g\left(u_{1}^{N}\right)=u_{1}^{N} \text { for all } g \in G, \\
& \mathbf{s}\left(u_{2}^{N}\right)=-u_{2}^{N}, \\
& \mathbf{t}\left(u_{2}^{N}\right)=\zeta_{3}^{2} u_{2}^{N} \text { and } \\
& \mathbf{u}\left(u_{2}^{N}\right)=u_{2}^{N} .
\end{aligned}
$$

The resulting $G$-module structure on $U_{\sigma}^{N}$ yields the direct sum of the trivial representation with the unique 1-dimensional representation of $G$ with fake degree $q^{22}$, which is $G$-isomorphic to the $\mathbb{C}$-span of the $G$-harmonic semi-invariant homogeneous polynomial

$$
a_{22}^{H}:=\left(x^{21} y-x y^{21}\right)+27\left(x^{17} y^{5}-x^{5} y^{17}\right)+170\left(x^{13} y^{9}-x^{9} y^{13}\right) \in \mathcal{C}_{G} \subset S\left(V^{*}\right),
$$

so that a basis for $\left(\mathcal{C}_{G} \otimes\left(U_{\sigma}^{N}\right)^{*}\right)^{G}$ is given by $\left\{1 \otimes u_{1}^{N}, a_{22}^{H} \otimes u_{2}^{N}\right\}$.

On the other hand, the $H$-module structure on $U_{\sigma}^{N}$ yields the direct sum of the trivial representation with the 1-dimensional representation of $H$ with fake degree $q^{3}$ given by the $\mathbb{C}$-span of the $H$-harmonic semi-invariant $\mathbf{N}$-homogeneous polynomial

$$
a_{22}^{H}=N_{1} N_{2}^{2} \in \mathcal{C}_{H} \subset S\left(E^{*}\right),
$$

so that a basis for $\left(\mathcal{C}_{H} \otimes\left(U_{\sigma}^{N}\right)^{*}\right)^{H}$ is given by $\left\{1 \otimes u_{1}^{N}, N_{1} N_{2}^{2} \otimes u_{2}^{N}\right\}$.

Thus, we witness the general isomorphism

$$
\left(\mathcal{C}_{G} \otimes\left(V^{\sigma}\right)^{*}\right)^{G} \simeq\left(\mathcal{C}_{H} \otimes\left(U_{\sigma}^{N}\right)^{*}\right)^{H}
$$

from the proof of Corollary 3.7 in this example, because we obtain $a_{22}^{H} \otimes u_{2}^{N} \mapsto u_{2}^{G}$ by collapsing the first tensor in

$$
\begin{aligned}
a_{22}^{H} \otimes u_{2}^{N} & =\left(x^{5} y-x y^{5}\right)\left(x^{8}+14 x^{4} y^{4}+y^{8}\right)^{2} \otimes\left(y \otimes x^{\sigma}-x \otimes y^{\sigma}\right) \in\left(\mathcal{C}_{H} \otimes\left(U_{\sigma}^{N}\right)^{*}\right)^{H} \\
& \mapsto\left(x^{5} y-x y^{5}\right)\left(x^{8}+14 x^{4} y^{4}+y^{8}\right)^{2}\left(y \otimes x^{\sigma}-x \otimes y^{\sigma}\right)=u_{2}^{G} \in\left(\mathcal{C}_{G} \otimes\left(V^{\sigma}\right)^{*}\right)^{G}
\end{aligned}
$$

Acknowledgements We thank Theo Douvropoulos for many helpful remarks and suggestions. C. Arreche was partially supported by NSF grant CCF-1815108. N. Williams was partially supported by Simons Foundation award number 585380 .

\section{References}

[1] C. Arreche and N. Williams, Normal reflection subgroups, Proc. FPSAC 2020, Sém. Lothar. Combin. 84B (2020), Art. 92.

[2] C. Arreche and N. Williams, Normal reflection subgroups, https://cocalc.com/share/ 67b57b8d53e5ef21bd2dcb88ea7ece7ccfee23e2/Normal/\%20Reflection/\%20Subgroups. sagews? viewer $=$ share, 2020 (accessed 26 June, 2020).

[3] H. Barcelo and A. Goupil, Combinatorial aspects of the Poincaré polynomial associated with a reflection group, Contemp. Math. 178 (1994), 21-43.

[4] D. Bessis, C. Bonnafé and R. Rouquier, Quotients et extensions de groupes de réflexion, Math. Ann. 323(3) (2002), 405-436. 
[5] A. Buorner and F. Brenti, Combinatorics of Coxeter Groups, Graduate Texts in Mathematics, Vol. 331 (Springer Science \& Business Media, New York, 2006).

[6] C. Bonnafé and M. J. Dyer, Semidirect product decomposition of Coxeter groups, Comm. Algebra 38(4) (2010), 1549-1574.

[7] C. Bonnafé, G. I. Lehrer and J. Michel, Twisted invariant theory for reflection groups, Nagoya Math. J. 182 (2006), 135-170.

[8] C. Chevalley, The Betti numbers of the exceptional simple Lie groups, in Proceedings of the International Congress of Mathematicians, Vol. 2 (American Mathematical Society, Providence, RI, Cambridge, MA, 1950), pp. 21-24.

[9] C. Chevalley, Invariants of finite groups generated by reflections, Amer. J. Math. 77(4) (1955), 778-782.

[10] H. S. M. CoxeTER, The product of the generators of a finite group generated by reflections, Duke Math. J. 18(4) (1951), 765-782.

[11] Ś. GAL, On normal subgroups of Coxeter groups generated by standard parabolic subgroups, Geom. Dedicata 115(1) (2005), 65-78.

[12] H. Hopf, Über die Topologie der Gruppen-Mannigfaltigkeiten und ihrer Verallgemeinerungen, in Selecta Heinz Hopf (Springer, Springer-Verlag, Berlin, New York, 1964), pp. 119151.

[13] J. E. Humphreys, Reflection Groups and Coxeter Groups, Cambridge Studies in Advanced Mathematics, Vol. 29 (Cambridge University Press, Cambridge, UK, 2009).

[14] G. I. Lehrer and D. E. Taylor, Unitary Reflection Groups, Austral. Math. Soc. Lect. Series, Vol. 20 (Cambridge University Press, New York, 2009).

[15] I. MARin AND J. Michel, Automorphisms of complex reflection groups, Represent. Theory 14 (2010), 747-788.

[16] P. Orlik And L. Solomon, Unitary reflection groups and cohomology, Invent. Math. 59(1) (1980), 77-94.

[17] M. REEDER, On the cohomology of compact Lie groups, Enseign. Math. 41(2) (1995), $181-200$.

[18] V. Reiner And A. V. Shepler, Invariant derivations and differential forms for reflection groups, Proc. London Math. Soc. 119(2) (2019), 329-357.

[19] G. C. Shephard and J. A. Todd, Finite unitary reflection groups, Can. J. Math. 6(2) (1954), 274-301.

[20] L. Solomon, Invariants of finite reflection groups, Nagoya Math. J. 22 (1963), 57-64.

[21] The Sage Developers, Sagemath, the Sage Mathematics Software System (Version 8.6), 2018, https://www.sagemath.org.

[22] N. Williams, Reflexponents, Proc. Amer. Math. Soc. 148 (2020), 3685-3698. 